\title{
THALAMOCORTICAL AND THE DUAL PATTERN OF CORTICOTHALAMIC PROJECTIONS OF THE POSTERIOR PARIETAL CORTEX IN MACAQUE MONKEYS
}

\author{
C. CAPPE, ${ }^{\mathrm{a}, \mathrm{b}}$ A. MOREL ${ }^{\mathrm{c}}$ AND E. M. ROUILLER ${ }^{\mathrm{a} *}$ \\ a Unit of Physiology and Program in Neurosciences, Department of \\ Medicine, Faculty of Sciences, University of Fribourg, Rue du Musée \\ 5, CH-1700 Fribourg, Switzerland \\ ${ }^{b}$ Centre de Recherche Cerveau et Cognition (UMR 5549) CNRS, \\ Université Paul Sabatier Toulouse 3, Faculté de Médecine de \\ Rangueil, 31062 Toulouse Cedex 9, France \\ ${ }^{c}$ Department of Functional Neurosurgery, Neurosurgery Clinic, Univer- \\ sity Hospital Zürich, Sternwartstrasse 6, CH-8091 Zürich, Switzerland
}

\begin{abstract}
The corticothalamic projection includes a main, modulatory projection from cortical layer VI terminating with small endings whereas a less numerous, driving projection from layer $\mathbf{V}$ forms giant endings. Such dual pattern of corticothalamic projections is well established in rodents and cats for many cortical areas. In non-human primates (monkeys), it has been reported for the primary sensory cortices (A1, V1, S1), the motor and premotor cortical areas and, in the parietal lobe, also for area 7 . The present study aimed first at refining the cytoarchitecture parcellation of area 5 into the sub-areas PE and PEa and, second, establishing whether area 5 also exhibits this dual pattern of corticothalamic projection and what is its precise topography. To this aim, the tracer biotinylated dextran amine (BDA) was injected in area $P E$ in one monkey and in area PEa in a second monkey. Area PE sends a major projection terminating with small endings to the thalamic lateral posterior nucleus (LP), ventral posterior lateral nucleus (VPL), medial pulvinar (PuM) and, but fewer, to ventral lateral posterior nucleus, dorsal division (VLpd), central lateral nucleus (CL) and center median nucleus (CM), whereas giant endings formed restricted terminal fields in LP, VPL and PuM. For area PEa, the corticothalamic projection formed by small endings was found mainly in LP, VPL, anterior pulvinar (PuA), lateral pulvinar (PuL), PuM and, to a lesser extent, in ventral posterior inferior nucleus (VPI), CL, mediodorsal nucleus (MD) and CM. Giant endings originating from area PEa formed restricted terminal fields in LP, VPL, PuA, PuM, MD and PuL. Furthermore, the origin of the thalamocortical projections to areas PE and PEa was established, exhibiting clusters of neurons in the same thalamic nuclei as above, in other words predominantly in the caudal
\end{abstract}

*Corresponding author. Tel: +41-26-300-86-09; fax: +41-26-300-96-75. E-mail address: Eric.Rouiller@unifr.ch (E. M. Rouiller).

Abbreviations: AChE, acetylcholinesterase; BDA, biotinylated dextran amine; $\mathrm{CL}$, central lateral nucleus; $\mathrm{CM}$, center median nucleus; $\mathrm{CT}$, corticothalamic; FEF, frontal eye field; FR, Fluro-Ruby; IPS, intraparietal sulcus; IT, infero-temporal area; LP, lateral posterior nucleus; MD, mediodorsal nucleus; MK1, monkey 1 ; MK2, monkey 2; PMd-c, caudal part of dorsal premotor cortex; PMd-r, rostral part of dorsal premotor cortex; PMv-c, caudal part of ventral premotor cortex; PMv-r, rostral part of ventral premotor cortex; $\mathrm{Pu}$, pulvinar nucleus; $\mathrm{PuA}$, anterior pulvinar; PuL, lateral pulvinar; PuM, medial pulvinar; TC, thalamocortical; VA, ventral anterior nucleus; VLa, ventral lateral anterior nucleus; VPI, ventral posterior inferior nucleus; VPL, ventral posterior lateral nucleus; WGA, wheat germ agglutinin. thalamus. Via the giant endings CT projection, areas PE and PEa may send feedforward, transthalamic projections to remote cortical areas in the parietal, temporal and frontal lobes contributing to polysensory and sensorimotor integration, relevant for visual guidance of reaching movements for instance.

Key words: parietal lobe, primate, thalamus, retrograde and anterograde tracing, axon terminal, BDA.

The posterior parietal cerebral cortex is a key region where different sensory modalities interact, representing a polymodal basis for programming goal-directed action by cooperation with the frontal lobe (prefrontal and premotor cortical areas). Although such cortico-cortical connections may be the main substrate for polysensorimotor integration, one may consider the possibility of complementary contributions involving subcortical levels. In this context, the thalamus is a possible candidate considering its strong input-output connections with multiple cortical areas, both sensory and motor. The present report is part of a broader study aimed at assessing the degree of overlap versus segregation of thalamic territories projecting to multiple cortical areas of the parietal, temporal and frontal lobes, involved in multisensory and sensori-motor integration. To this aim, injections of up to seven neuroanatomical tracers were performed in two monkeys in various areas of the posterior parietal cortex, auditory cortex and premotor cortex to retrogradely label the corresponding thalamocortical (TC) neurons (see Cappe et al., 2005 for a preliminary report). These data based on multiple retrograde tracing will be reported in detail elsewhere. The present report focuses on the analysis of data derived from one of these tracers, biotinylated dextran amine (BDA), yielding not only retrograde labeling in thalamus as the other tracers but, even more prominently, anterograde labeling. BDA was injected in two monkeys in the posterior parietal associative cortex, thus allowing us to study in detail the pattern of its TC and corticothalamic (CT) projections.

The CT projection is known to be organized in mammals according to a dual pattern (see Giguere and GoldmanRakic, 1988; Schwartz et al., 1991 for the prefrontal cortex in macaque monkeys; see Kelly and Wong, 1981; Ojima, 1994 for the primary auditory cortex of the cat; see Kakei and Shinoda, 2001 for the motor cortex of the cat; see Bourassa et al., 1995 for SI in the rat; see Darian-Smith et al., 1999 for the somatosensory cortex of the macaque monkey; see Bourassa and Deschênes, 1995 for the 
primary visual cortex of the rat). First, the major CT projection originates from cortical layer $\mathrm{VI}$, forming dense and diffuse terminal fields of small endings, functionally corresponding to a feedback "modulatory" projection from cerebral cortex onto thalamus. Second, a quantitatively less abundant CT projection originates from cortical layer $V$ and terminates in the thalamus with so-called giant endings. Functionally, this second CT projection is hypothesized to correspond to a feedforward "driving" projection, through which two remote cortical areas are connected via the thalamus (e.g. Guillery, 1995). This dual pattern of CT projection is maintained across species (rodents, cats, monkeys) as well as across sensory and motor systems (see Rouiller and Welker, 2000 for review).

More specifically, this dual pattern of CT projection has been described in much detail for primary cortical areas in rodents, such as the primary visual cortex (Bourassa and Deschênes, 1995), primary somatosensory cortex (Hoogland et al., 1987, 1991; Welker et al., 1988; Bourassa et al., 1995), primary auditory cortex (Rouiller and Welker, 1991) and primary motor cortex (Rouiller et al., 1991). Later, evidence for such dual pattern of CT projection was extended to the cat (auditory system: Ojima, 1994; Bajo et al., 1995; Winer et al., 1999; motor system: Kakei et al., 2001; visual system: Ojima et al., 1996), with extension in some cases to non primary areas. As far as primates are concerned, the dual CT projection has been demonstrated for the visual cortex (Rockland, 1996), motor cortex (Rouiller et al., 1998, 2003), auditory cortex (Rouiller and Durif, 2004) and prefrontal cortex (Schwartz et al., 1991). In the parietal lobe, a dual CT projection has been reported in monkeys for areas $1,3 \mathrm{~b}$ and $7 \mathrm{a}$ (Darian-Smith et al., 1999) as well as for areas PFG and Opt in the caudal part of the lower bank of intraparietal sulcus (IPS) (Taktakishvili et al., 2002). The BDA injections performed in the present study aim at extending these data to area 5 of the posterior parietal cortex of macaque monkeys. In some previous studies (Sakata et al., 1973; Pearson and Powell, 1978), area 5 was considered as a single entity whereas in other studies (Pandya and Seltzer, 1982; Matelli et al., 1998) area 5 was subdivided in two parts, namely area PE (also referred to as area $5 \mathrm{~d}$ ) and area PEa (named also area $5 \mathrm{v}$ ). In the present study, we re-examined the cytoarchitectonic characteristics of areas PE and PEa on the basis of Nissl staining and immunoreactivity to the non-phosphorylated neurofilament protein SMI-32. Furthermore, we addressed the issue of whether PE and PEa differ with respect to their TC and CT connections. To these aims, BDA injections were placed in one monkey, in the superior parietal lobule, in caudal area 5 in a zone presumably corresponding to area PE and in a second monkey, in the rostral (upper) bank of IPS, a region presumably corresponding to area $\mathrm{PEa}$. Besides the type of pattern of CT projection (small versus giant endings), the topography of the CT projection assessed here with BDA will be compared with previous descriptions (Yeterian and Pandya, 1985; Acuna et al., 1990 ) based on anterograde tracing methods (autoradiography, wheat germ agglutinin conjugated to horseradish peroxidase (WGA-HRP)) which revealed the topography but not the differential fine morphology of CT endings. Moreover, BDA also generates some retrograde labeling, offering the possibility to compare the distribution of TC neurons projecting to area 5 with previous studies (Pearson and Powell, 1978; Jones et al., 1979; Miyata and Sasaki, 1983; Acuna et al., 1990; Avendano et al., 1990). Finally, in the same two monkeys, additional TC data were derived from injections of other retrograde tracers in areas PEa and PE.

\section{EXPERIMENTAL PROCEDURES}

The present study was conducted on two adult macaque monkeys (one Macaca mulatta and one Macaca fascicularis), 3 and 4 years old and weighing 3 and $4 \mathrm{~kg}$. Surgical procedures and animal care were conducted in accordance with the Guide for Care and Use of Laboratory Animals (ISBN 0-309-05377-3; 1996) and were approved by local (Swiss) veterinary authorities. Experimental procedures were designed to minimize the animals' pain and suffering.

\section{Tracer injections}

BDA (10,000 MW; Molecular Probes, Eugene, OR, USA), FluroRuby (FR; Molecular Probes) and wheat germ agglutinin (WGA; Sigma, Lyon, France) were injected at multiple sites in areas $\mathrm{PEa}$ and $\mathrm{PE}$ of the posterior parietal cortex. We injected also multiple retrograde tracers in other cortical areas in the temporal and frontal lobes, but these data will be described separately in another report.

These injections were performed following experimental procedures previously described in detail (Rouiller et al., 1998, 1999, 2003; Liu et al., 2002; Tanné-Gariépy et al., 2002). In all cases, sterile surgical procedures were followed. The monkeys were pre-anesthetized with ketamine $(5 \mathrm{mg} / \mathrm{kg}$, i.m.) and treated with the analgesic carprofen (Rymadil; $4 \mathrm{mg} / \mathrm{kg}$, s.c.), antibiotics (Albipen: ampicillin $10 \%, 15-30 \mathrm{mg} / \mathrm{kg}$, s.c.), atropine sulfate (0.05 ml/kg i.m.), and with dexamethasone (Decadron, $0.05 \mathrm{ml} / \mathrm{kg}$ diluted 1:1 in saline, i.m.). Then, monkeys were anesthetized with a continuous perfusion through the femoral vein $(0.1 \mathrm{ml} / \mathrm{min} / \mathrm{kg})$ of a mixture of propofol (1\%) and $4 \%$ glucose solution to which ketamine was added $(65 \mathrm{mg} / 100 \mathrm{ml})$. Animals were placed in a stereotaxic frame for surgery under aseptic conditions. The skull was opened above the parietal lobe on one side, exposing the IPS, which was used as landmark. The dura mater was dissected for visualization of the injection zone. A syringe (Hamilton, Reno, $\mathrm{NV}$, USA; 5 or $10 \mu \mathrm{l}$ ) was inserted perpendicularly to cortical surface and used for tracer injections. Detailed information on the location of injections, tracers injected and amounts delivered is given in Table 1 and the multiple sites of cortical injections for each tracer are illustrated in Fig. 2. After injections, the muscles and skin were sutured and the animal was treated during several days with an analgesic (pills of Rymadil mixed with food; $5 \mathrm{mg} / \mathrm{kg}$, p.o.) and an antibiotic (amoxicillin; $10 \mathrm{mg} / \mathrm{kg}$, p.o.). Injections of tracers were performed 2-3 weeks before the animals were killed. After the survival period, each monkey was pre-anesthetized with ketamine $(5 \mathrm{mg} / \mathrm{kg}$, i.m. $)$ and given an overdose of sodium pentobarbital (Vetanarcol; $90 \mathrm{mg} / \mathrm{kg}$, i.p.). Transcardiac perfusion of

Table 1. Summary of injection sites and tracers

\begin{tabular}{lllll}
\hline Monkey & Areas injected & Tracers & $\begin{array}{l}\text { Number of } \\
\text { injections }\end{array}$ & $\begin{array}{l}\text { Total amount } \\
\text { injected }(\mu \mathrm{l})\end{array}$ \\
\hline MK1 & Areas PEa/PE & FR/BDA & $7 / 6$ & $2.8 / 6$ \\
MK2 & Areas PEa/PE & BDA/WGA & $7 / 6$ & $6 / 2.4$ \\
\hline
\end{tabular}


saline $(0.9 \%)$ was followed by paraformaldehyde $(4 \%$ in phosphate buffer $0.1 \mathrm{M}, \mathrm{pH} 7.4$ ), and 10,20 and $30 \%$ solutions of sucrose in phosphate buffer. The brain was dissected and stored overnight in a solution of $30 \%$ sucrose in phosphate buffer.

\section{Histological processing}

Frozen sections were cut in the frontal plane (40 $\mu \mathrm{m}$ thick) with a cryostat and collected in five series. One series was immediately mounted on slides and stored at $4{ }^{\circ} \mathrm{C}$ for analysis of the fluorescent tracers. One section out of two mounted for fluorescence analysis was later counterstained for Nissl with Cresyl Violet. Other series were stored in $0.1 \mathrm{M}$ phosphate buffer at $4{ }^{\circ} \mathrm{C}$ and later processed to visualize BDA or WGA labeling (as previously described in detail in Rouiller et al., 1998, 1999, 2003) as well as for acetylcholinesterase (AChE; Geneser-Jensen and Blackstad, 1971). The labeling was examined with an Olympus light microscope (Olympus, Tokyo, Japan). In the thalamus, retrogradely labeled TC neurons were plotted on sections taken at $0.2 \mathrm{~mm}$ intervals using Neurolucida (MicroBrightField, Inc., Colchester, VT, USA). The anterogradely BDA-labeled terminals fields, corresponding to dense axonal arborizations with boutons en passant and terminaux, were also charted with Neurolucida. From the same BDA sections, the precise locations of giant endings and of retrogradely BDA-labeled neurons were also charted. Drawings with plots of labeled cells and terminal fields were then exported in the form of computer files formatted for later processing using the software CorelDraw 12 or Adobe Illustrator 10.

In the two monkeys subjected to tracer injections (Table 1), all series of sections were used to visualize the cells labeled with the various tracers and to assess the parcellation of the thalamus using AChE and Nissl staining. In the same two monkeys, Nissl staining was also used to identify areas PE and PEa using previously established criteria (Pandya and Seltzer, 1982). To better differentiate areas PE and PEa, sections immunostained for SMI-32 (Campbell and Morrison, 1989; Tsang et al., 2000) derived from two other monkeys were analyzed. To reveal SMI-32, sections were processed immunocytochemically as previously described (Liu et al., 2002; Wannier et al., 2005).

\section{Data analysis}

Contours of Nissl-stained sections were drawn with the aid of a drawing tube, and then adjacent sections stained for AChE were superimposed using contours and blood vessels. These drawings were scanned and imported in Neurolucida software and then aligned to sections containing plots of fluorescent, WGA or BDA labeling. The distribution of AChE, in addition to the Nissl staining provided means for refined parcellation of thalamic nuclei (Fig. 5; see also Morel et al., 2005).

In the present report, we first analyzed the location of anterogradely BDA-labeled axon terminal fields formed by small and/or giant endings. Second, the distribution of retrogradely labeled cells was assessed, to be confronted with the distribution of anterograde labeling. Furthermore, retrograde labeling was analyzed quantitatively as follows:

a) The relative contribution of inputs from different thalamic nuclei to areas PEa and PE was assessed by counting, for each tracer injected, the number of labeled cells in each thalamic nucleus and calculating the percentage of the total number of cells labeled with this particular tracer in the thalamus. This quantitative analysis included the entire rostro-caudal extent of thalamus with a regular interval between consecutive sections analyzed and, for each of them, the entire extent of each thalamic nucleus was systematically scanned for detection of retrogradely labeled neurons. Such a procedure does not correspond to a strict stereological method and therefore possible sampling bias cannot be excluded. However, our analysis was based on relative numbers (percentage of retrogradely labeled neurons in each thalamic nucleus with respect to the total number of labeled neurons in thalamus) and possible bias will thus affect all thalamic nuclei in a similar manner. As a consequence, our comparisons of relative numbers of TC neurons across thalamic nuclei projecting to the different cortical areas remain valid. A similar method has been used to compare the relative strength of thalamic inputs to different cortical areas (e.g. Matelli et al., 1989; Darian-Smith et al., 1990; Hatanaka et al., 2003; Morel et al., 2005). Moreover, as the number of labeled neurons in thalamus was relatively low, the use of a stereological probe to estimate their number was not appropriate and therefore we counted all labeled neurons. This approach is considered as adequate as all samples were analyzed using the same procedure (Lavenex et al., 2000; Geuna, 2000; Benes and Lange, 2001).

b) Another analysis aimed at measuring the degree of segregation versus overlap between subpopulations of neurons labeled by injections of tracers in areas PEa and PE. An index of overlap was calculated according to the method described by TannéGariépy et al. (2002; see also Morel et al., 2005). In brief, on each section of thalamus analyzed, a grid of $0.5 \times 0.5 \mathrm{~mm}$ units was aligned to the medial thalamic border. Then, the following counts were made for the whole thalamus, without considering thalamic nuclear boundaries: (i) number of square units containing only neurons labeled with the first tracer (N1), (ii) number of square units containing only neurons labeled with the second tracer (N2) and (iii) number of square units containing neurons labeled with one or the other tracer (N3). The N1, N2 and N3 values were averaged over all or part of the thalamus. A mean "global" index of overlap was calculated from $[\mathrm{N} 3 /(\mathrm{N} 1+\mathrm{N} 2+\mathrm{N} 3) \times 100]$. A value of $0 \%($ with $\mathrm{N} 3=0)$ corresponds to a complete segregation of neurons labeled by each tracer, whereas a mean value of $100 \%(\mathrm{~N} 3=\mathrm{N}$ tot, with $\mathrm{N} 1=0$ and $\mathrm{N} 2=0$ ) corresponds to a complete overlap between the two populations of labeled neurons. This procedure was applied for the combination of tracers FR and BDA in monkey 1 (MK1) and the combination of tracers WGA and BDA in monkey 2 (MK2).

\section{RESULTS}

\section{Localization of injections sites}

Area PE occupies the surface of rostral superior parietal lobule whereas area PEa is located in the upper bank of the IPS (Paxinos et al., 2000). The distinction between areas PE and PEa is based on cytoarchitectonic criteria as initially defined in Nissl material by Pandya and Seltzer (1982) and reported later in other studies (Seltzer and Pandya, 1984, 1986; Matelli et al., 1998). In line with these data (Pandya and Seltzer, 1982), Niss--stained sections adjacent to the injection sites in the two monkeys (Fig. 1) allowed us to confirm that cortical layer II of area PE is weakly differentiated, whereas layer III appears broad and clearly distinct from the adjacent layers II and IV. Layer V in PE contains sparse medium-sized cells, thought not completely segregated from a modest layer VI (Fig. 1B). Confirming previous data (Pandya and Seltzer, 1982), area PEa can be distinguished from area PE by its less prominent layer IV but, on the contrary, by a more developed and better defined layer VI (Fig. 1C). As observed in two separate monkeys (see Experimental Procedures section), immunostaining for SMI-32 provided additional criteria to distinguish area PE from area PEa (Fig. 1D, E and F). Area $P E$ is characterized by denser staining of pyramidal cells in layer III (Fig. 1E), whereas layer III in area PEa is less densely stained and is formed by smaller cells (Fig. $1 F)$. Layer $V$ of area $P E$ is well defined with sparse, large 

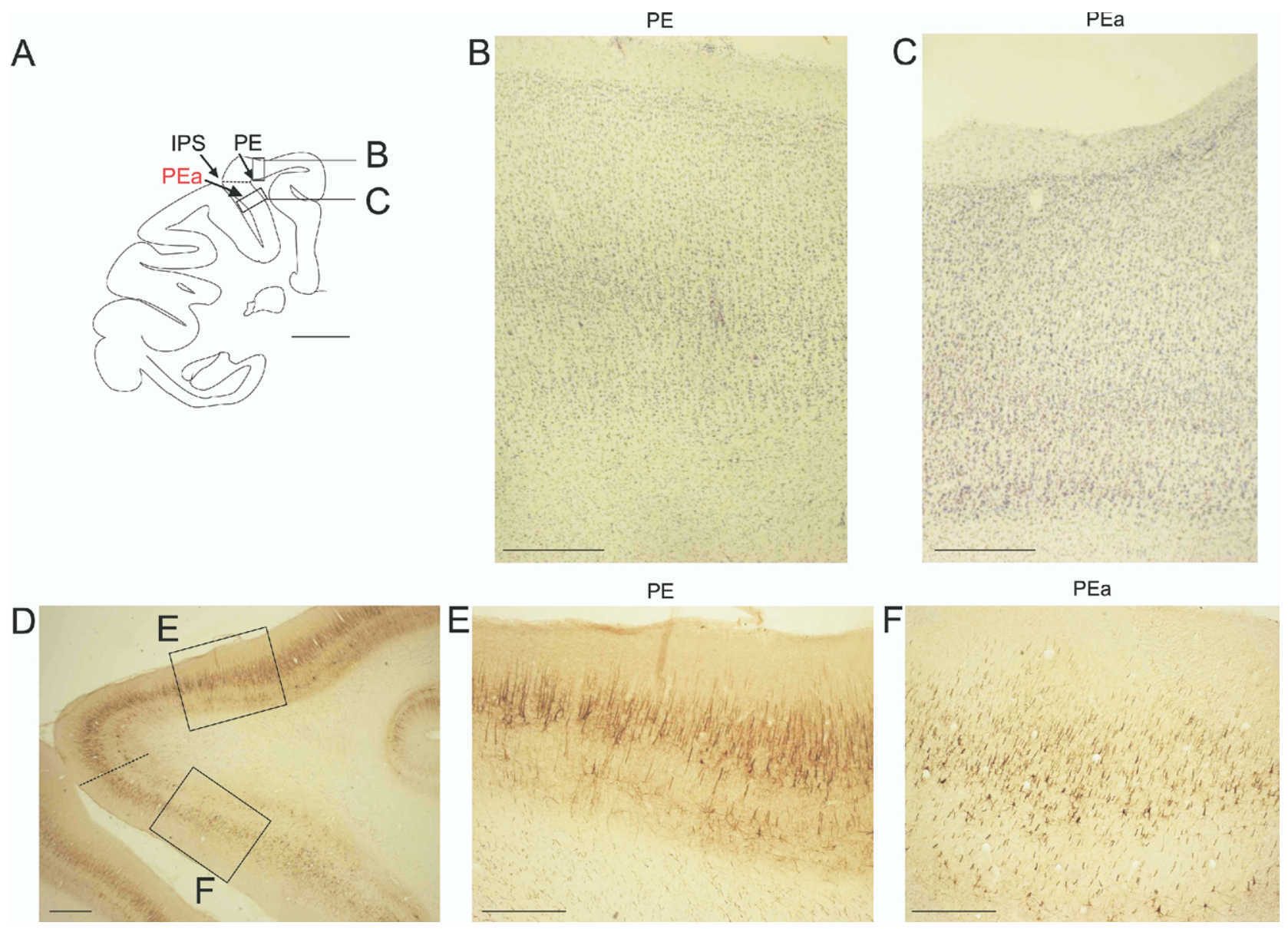

Fig. 1. Cytoarchitecture of areas PE and PEa. The localization of areas PE and PEa is indicated in panel $A$ on a frontal section of the left hemisphere of the macaque monkey, with two rectangles pointing to the zones shown by photomicrographs of Nissl staining at higher magnification in panels $B$ (for $\mathrm{PE}$ ) and $\mathrm{C}$ (for PEa). Panel D shows areas PE and PEa on a frontal section immunostained for SMI-32 with two rectangles painting to the location of the zones shown at higher magnification illustrated in panels $E$ and F. (A) Scale bar=10 mm. (B, C) Scale bar=500 $\mu \mathrm{m}$. (D-F) Scale bar=1 mm.

pyramidal cells (Fig. 1E), whereas layer $\mathrm{V}$ in area $\mathrm{PEa}$ is less densely stained with SMI-32 (Fig. 1F). SMI-32-stained material exhibits a rather clear transitional zone between areas PE and PEa, as indicated by the dashed line in Fig. 1D. A corresponding transitional zone can be seen in Nissl material, but less distinctly. Nevertheless, based on the Nissl staining in the two experimental monkeys, the injections aimed for areas PE and PEa, respectively (see below), were indeed located in the aimed areas (Fig. 2).
In each of two monkeys, we placed injections at multiple sites in the anterior bank of IPS, in one case BDA (MK1) and in the other case WGA (MK2), aiming at area PEa. In the same two monkeys, we injected also the superior parietal lobule, FR in MK1 and BDA in MK2, in an area corresponding to $\mathrm{PE}$. The multiple injection sites are reconstructed and illustrated in Fig. 2 and a summary of the parameters of injection is listed in Table 1. As illustrated by the serial reconstruction of the multiple injection

Abbreviations used in the figures

$\begin{array}{ll}\text { AD } & \text { anterodorsal nucleus } \\ \text { AM } & \text { anteromedial nucleus } \\ \text { AV } & \text { anteroventral nucleus } \\ \text { CeM } & \text { central medial nucleus } \\ \text { Hb } & \text { habenular nucleus } \\ \text { LD } & \text { lateral dorsal nucleus } \\ \text { LGN } & \text { lateral geniculate nucleus } \\ \text { Li } & \text { limitans nucleus } \\ \text { MGN } & \text { medial geniculate nucleus } \\ \text { MTT } & \text { mammillothalamic tract } \\ \text { Pf } & \text { parafascicular nucleus } \\ \text { Po } & \text { posterior nucleus }\end{array}$

$R \quad$ reticular nucleus of the thalamus

Rh rhomboid nucleus

$\mathrm{Sg} \quad$ suprageniculate nucleus

VA ventral anterior nucleus

VLp ventral lateral posterior nucleus

VLpd ventral lateral posterior nucleus, dorsal division

VLpv ventral lateral posterior nucleus, ventral division

VM ventral medial nucleus

VPM ventral posterior medial nucleus

ZI zona incerta

$\mathrm{X}$ area $\mathrm{X}$ 
A

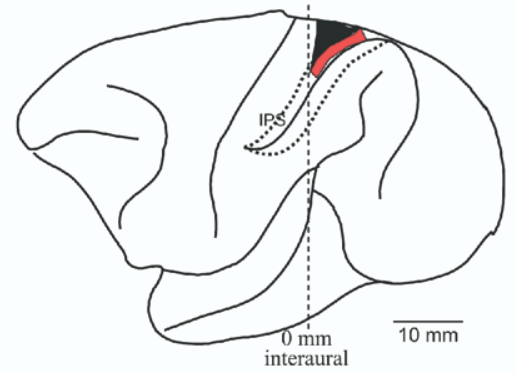

$\mathrm{C}$ Injection of FR in area PEa (MK1)

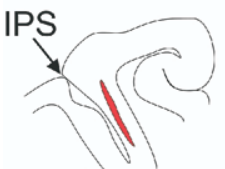

section 120
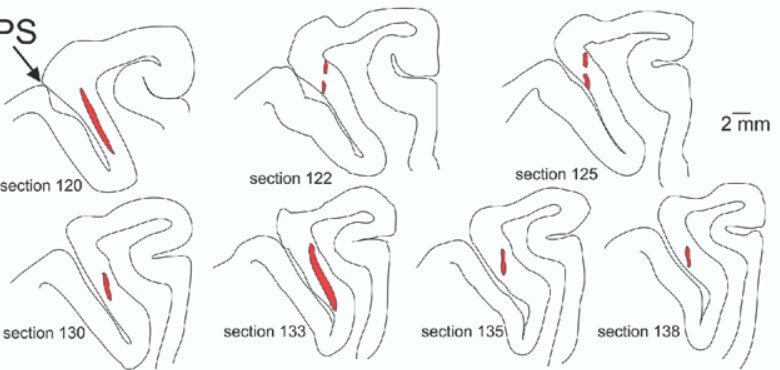

$\mathrm{E}$ Injection of BDA in area PEa (MK2)
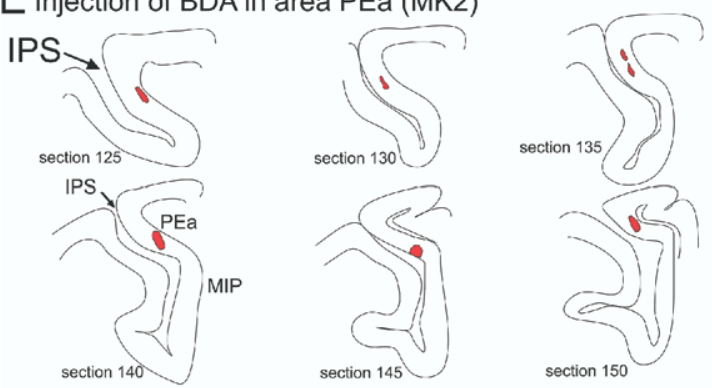

$G$ Injection of BDA in area PE (MK1)
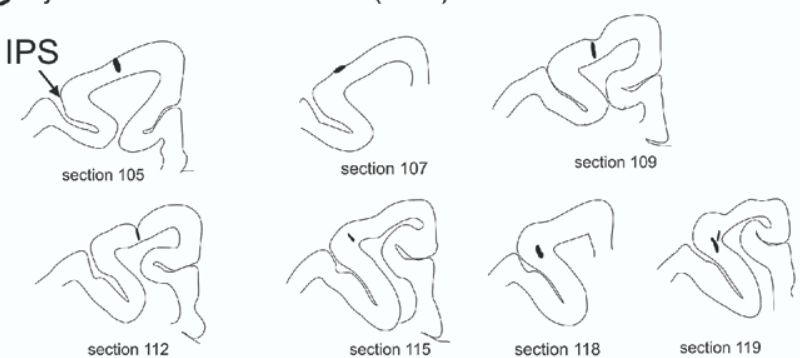

I Injection of WGA in area PE (MK2)

IPS

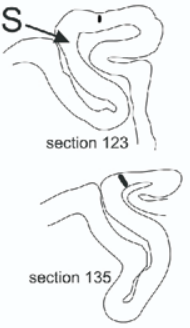

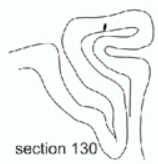

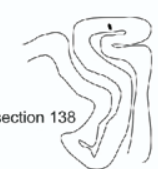

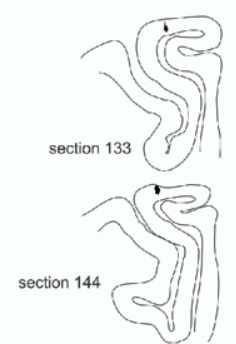

B

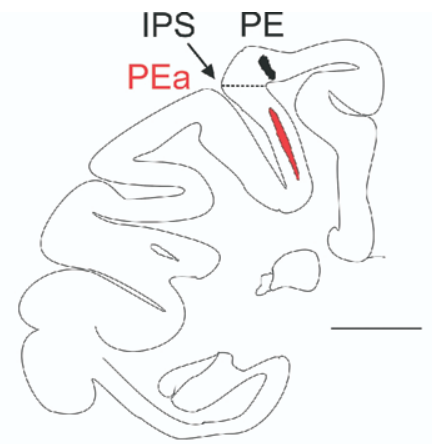

D

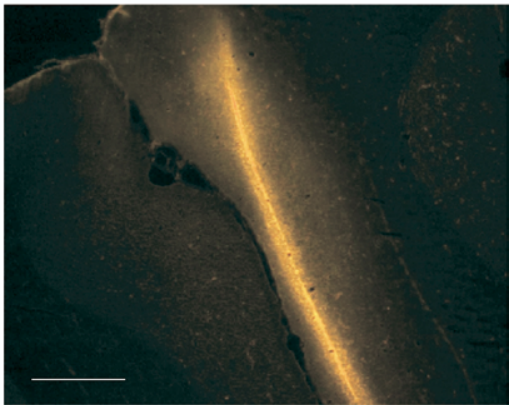

$\mathrm{F}$

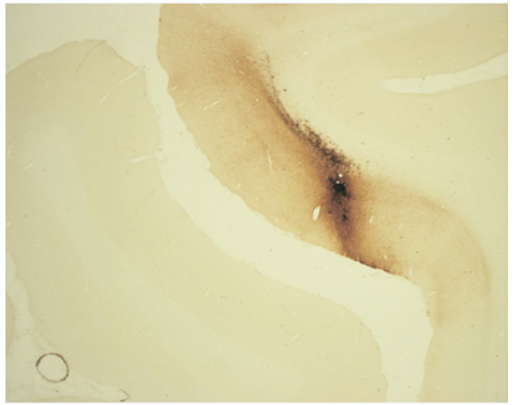

$\mathrm{H}$

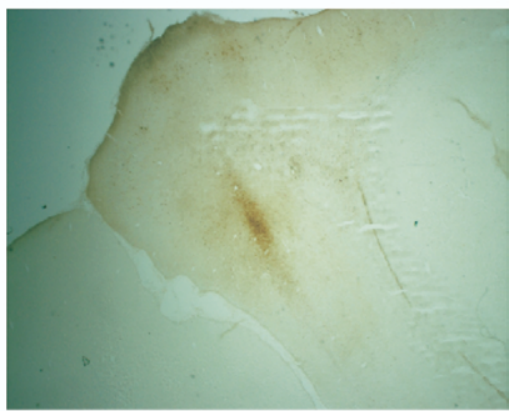

$\mathrm{J}$

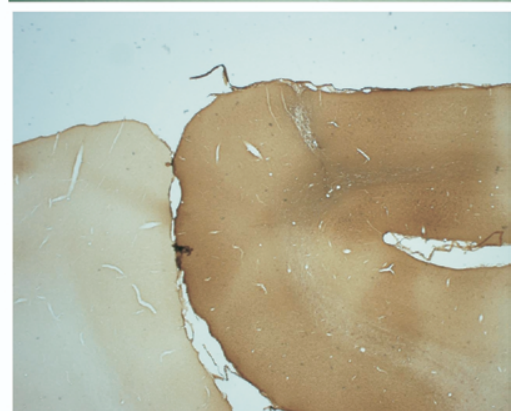

Fig. 2. Location of injection sites. Injections in area PE are indicated in black, injections in area PEa in red. The drawings in panels $A$ and $B$ show the location of injections on a lateral view and on a frontal section of the left hemisphere, respectively. Location and photomicrographs of the FR injection site in area PEa for MK1 (C and D), the BDA injection site in area PEa for MK2 (E and F), the BDA injection site in area PE for MK1 (G and H) and the WGA injection site in area PE for MK2 (I and J). The photomicrographs were taken in the center of the injection site. The multiple sites of injection were reconstructed for each tracer from several consecutive sections, arranged from rostral to caudal in left panels. (A, B) Scale bar=10 mm. 
sites on consecutive sections (Fig. 2C, E, G, I), the corresponding tracer spread on all cortical layers, in each cortical area injected. The total volume of cortical tissue injected with each tracer (the sum of all individual injection sites) covers a substantial portion of PE or PEa, certainly much bigger than a single cortical column.

\section{Thalamic parcellation}

The delimitation of thalamic nuclei and their nomenclature follow closely those introduced by Jones (Jones, 1985, 1990; Jones and Hendry, 1989). The distribution of AChE, in addition to Nissl staining, provided further criteria to assess nuclear borders, as illustrated for the caudal part of thalamus in Fig. 5. Differences in AChE densities particularly demarcate anterior pulvinar (PuA) and medial pulvinar (PuM) from ventral posterior lateral nucleus (VPL) laterally and center median nucleus (CM)-parafascicular nucleus (Pf) and central lateral nucleus (CL) medially, or from suprageniculate nucleus (SG)-limitans nucleus (Li) complex more caudally. Also inferior pulvinar (Pul) is clearly distinguished from adjacent lateral pulvinar (PuL) by enhanced AChE staining. In the anterior part of the thalamus, delimitation of motor nuclei, in particular area $X$ of Olszewski (1952) was also more reliably assessed by combining AChE and Nissl, as reported before (Morel et al., 2005).

\section{CT projections}

BDA is the anterograde tracer of choice to establish the CT projections originating from areas $\mathrm{PEa}$ and $\mathrm{PE}$, allowing identification of two types of CT terminals, corresponding to small ( $<2 \mu \mathrm{m}$ in diameter) and giant endings $(2-6 \mu \mathrm{m})$. The topographical distribution of BDA axonal terminal fields in the thalamus is shown in Figs. 3-6. Zones with small endings are indicated by yellow spots, whereas territories with giant endings are depicted by blue triangles. Typical CT axonal terminal fields formed by small endings or giant endings are illustrated in Fig. 6.

After BDA injection in area PE (MK1, Fig. 3; Fig. 5G and $\mathrm{H}$ ), the main $\mathrm{CT}$ projection formed by small endings terminated in lateral posterior nucleus (LP), VPL and PuM. Moreover, few additional clusters of small endings were found in ventral lateral posterior nucleus, dorsal division, $\mathrm{CL}$ and $\mathrm{CM}$. Giant CT endings were observed in LP, VPL and PuM. The injection of BDA in area PE revealed a gradient of labeling from rostral to caudal in the thalamus, with a more prominent labeling caudally.

After BDA injection in area PEa (MK2, Fig. 4; Fig. 5A, $C$ and $E$ ), the largest and densest CT terminal field formed by small endings was located in the thalamic nuclei LP, VPL, PuA, PuL and PuM. Additional, smaller and less dense clusters were found in ventral posterior inferior nucleus (VPI), CL, mediodorsal nucleus (MD) and CM. Giant CT endings were observed mainly in LP, VPL, PuA and PuM, with fewer, in MD and PuL. As for the injection in PE, the density of the labeling was less prominent in rostral thalamic nuclei like ventral anterior nucleus (VA) or ventral lateral anterior nucleus (VLa) than in caudal thalamic nuclei like LP or PuM. To illustrate more precisely the distribution of labeling caudally, additional intermediate sec- tions of the caudal thalamus are shown in Fig. 5 (panels A, $\mathrm{C}$ and $\mathrm{E}$ for MK2; panels $\mathrm{G}$ and $\mathrm{H}$ for MK1).

The respective distribution of the giant versus small $C T$ endings can be compared for MK2 in Figs. 4, 5 (A, C, E) and $6 \mathrm{~A}$. Clearly, the small CT endings (yellow areas) formed larger terminal fields than the areas where giant endings are present (blue triangles). There are widespread CT projection territories formed exclusively by small endings, representing in extent most of the CT terminal fields. Such large areas composed exclusively of small CT endings can be seen especially in LP (e.g. section 85 in Fig. 5A), PuA (section 87 in Fig. 4), VPL (section 89 in Fig. 5C and section 90 in Fig. 6) and in PuL (section 91 in Fig. 4). The CT terminal fields containing giant endings are, in nearly all cases, mixed terminal fields as they correspond to restricted territories where both types of terminals are present (see Fig. 6D and the overlap of blue triangles onto yellow areas in Figs. 4, 5 and 6). There is a general tendency for giant endings to be located at the periphery of the larger terminal fields formed by small endings. Typically, on a given thalamic section, giant endings are distributed into multiple small zones of projection, most often across distinct thalamic nuclei. For instance, in section 87 (Fig. 4), giant endings were seen in LP, PuA and VPL and, more caudally, in VPL, PuA and PuM (section 90 in Fig. 6). The CT projection formed by giant endings is restricted to multiple, small territories but widely dispersed across several thalamic nuclei, representing an important feature regarding their possible role for processing and transfer of information (see discussion). In MK1 (Fig. 3), although less clearly visible due to a generally less dense CT projection, high magnification drawings (not shown) confirmed these features, with a broader distribution of small CT endings than giant endings, the latter forming small territories overlapping the terminal fields formed by small endings at their periphery. Giant CT endings were seen mainly in LP and PuM in MK1.

\section{TC projections}

The distribution of retrogradely labeled neurons in thalamus after injections of tracers in areas PE and PEa is shown in Figs. 3 (for MK1), 4 (for MK2) and 5 (both monkeys). The consecutive frontal sections of thalamus show only sparse retrograde labeling rostrally, at levels of motor thalamic nuclei VA, VLa, ventral medial nucleus or $X$. In contrast, a high number of neurons projecting to area PEa were found in the caudal thalamic nuclei LP, VPL, PuM and PuL. This was also true, except for PuL, for the neurons projecting to area PE. Considering the whole thalamus, the labeling for $\mathrm{TC}$ projections to areas $\mathrm{PEa}$ and PE was densest in LP, VPL and PuM. For PuL, the retrograde labeling was dense only after injection in area $P E a$. A few BDA retrogradely labeled neurons are visible in panel C of Fig. 6 (arrows), whereas FR- and WGA-labeled TC neurons are illustrated in panels $E$ and F of Fig. 6.

More quantitatively, Fig. 7A and $7 \mathrm{C}$ shows that the majority of TC projections to area PEa originate from several thalamic nuclei such as LP (36\% of TC connections for MK1 and $42 \%$ for MK2), PuM (18\% for MK1 and 18\% for MK2), PuL (especially for MK1 with $27 \%$ of TC connec- 


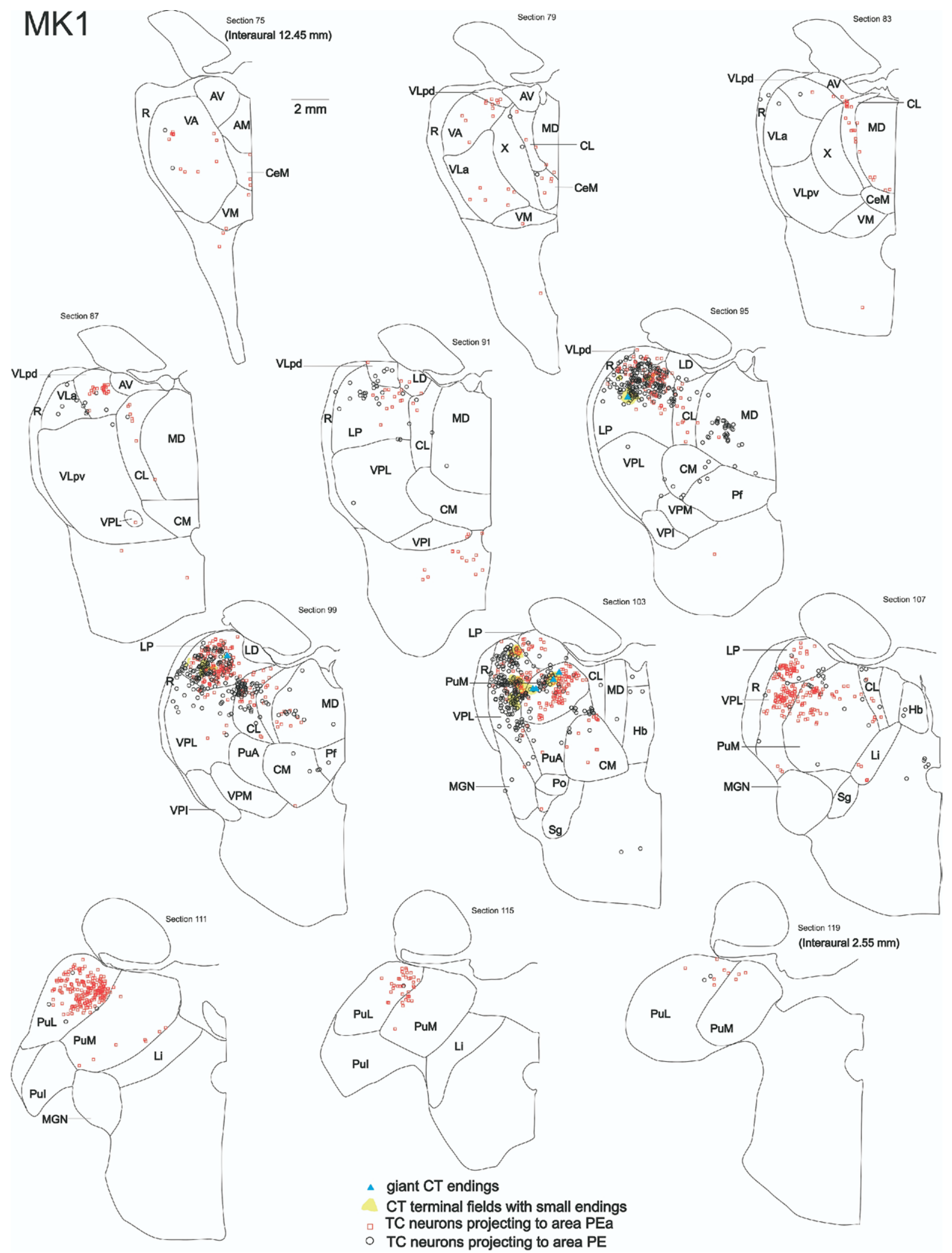

Fig. 3. Distribution of anterograde (small and giant CT endings) and retrograde (TC neurons) labeling in the thalamus, after injections of BDA (open black circles) and FR (open red squares) in areas PE and PEa, respectively, in MK1. The anterograde labeling (yellow areas and blue triangles) applies only for the BDA injection in area PE. Sections are arranged from rostral to caudal (75-119) and intervals between two consecutive sections are $0.2 \mathrm{~mm}$. Additional sections $(101,104)$ in the main zone of interest are shown for the same animal in Fig. 5 (panels $\mathrm{G}$ and $\mathrm{H}$ ). The corresponding stereotaxic levels (in mm) of monkey brain atlas (Paxinos et al., 2000) are indicated in parentheses for the most rostral and caudal sections. See list 


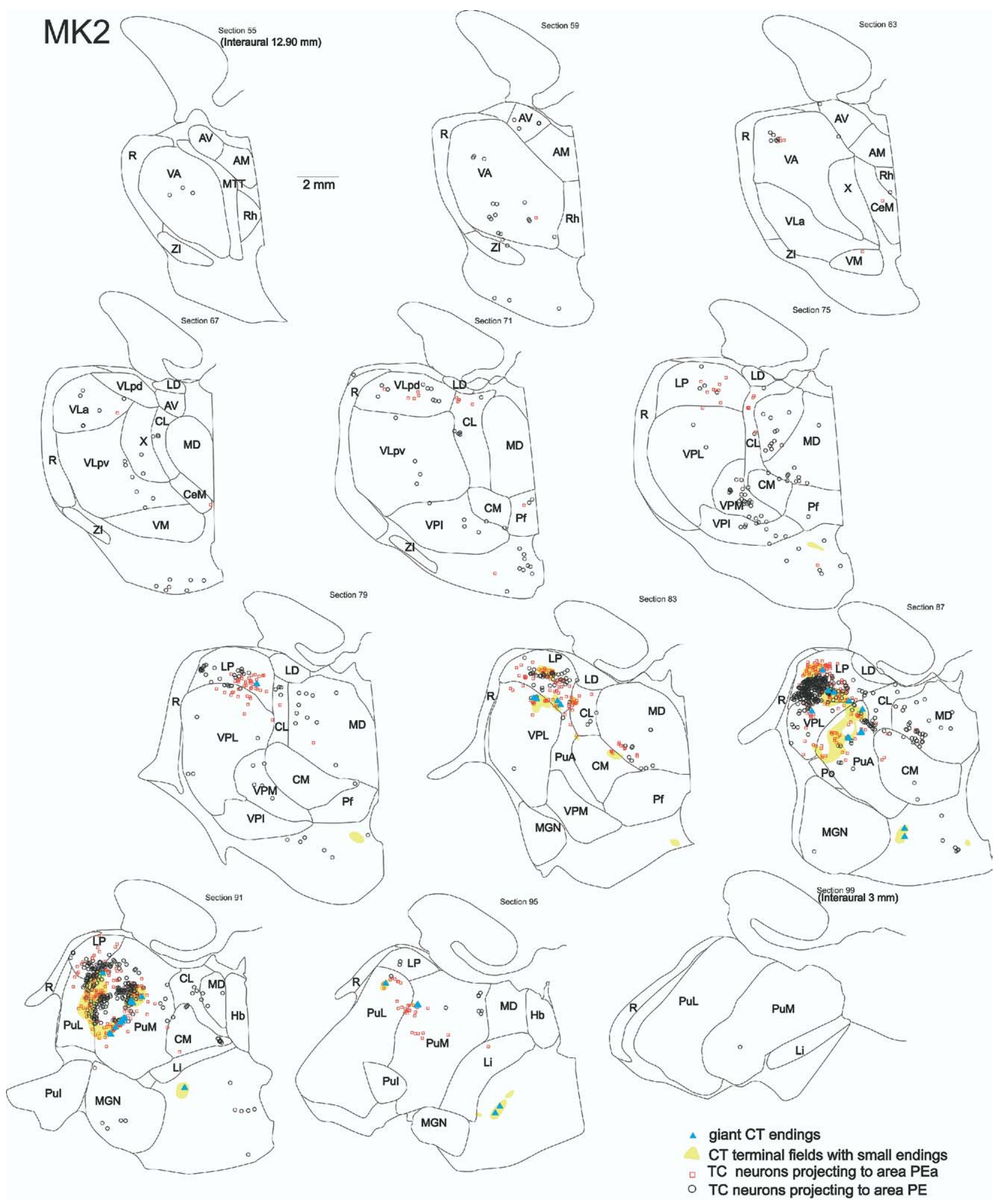

Fig. 4. Distribution of anterograde (small and giant CT endings) and retrograde (TC neurons) labeling in MK2 in the thalamus after injections of WGA (open black circles) and BDA (open red squares) in areas PE and PEa, respectively. The anterograde labeling applies only for the BDA injection in area PEa. Same conventions as in Fig. 3. Additional sections $(85,89$, and 93$)$ in the main zone of interest are shown for the same animal in Fig. 5 (panels $A, C$ and $E$ ).

tions, $3 \%$ only for MK2) and VPL (3\% for MK1 and 18\% for MK2). Less dense TC projections to area PEa originate from the thalamic nuclei CL, MD and PuA (for MK2). The $\mathrm{TC}$ projections to area $\mathrm{PE}$ are quantitatively shown in Fig. 
A

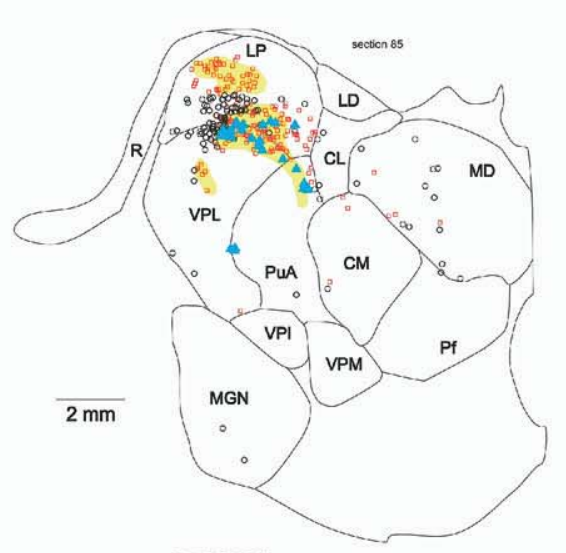

C

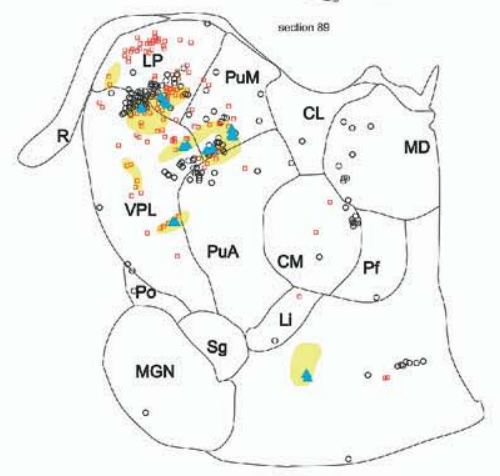

E

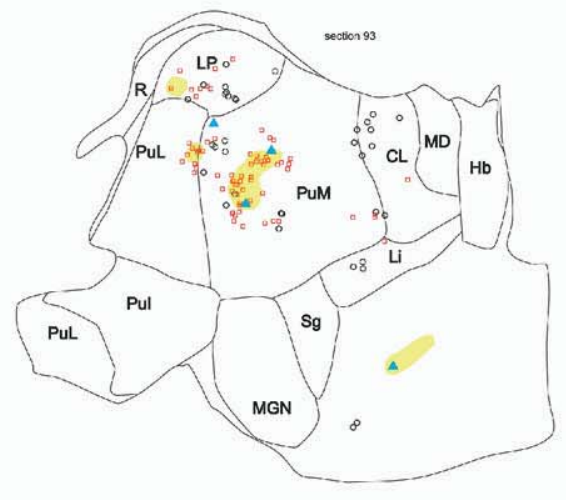

G

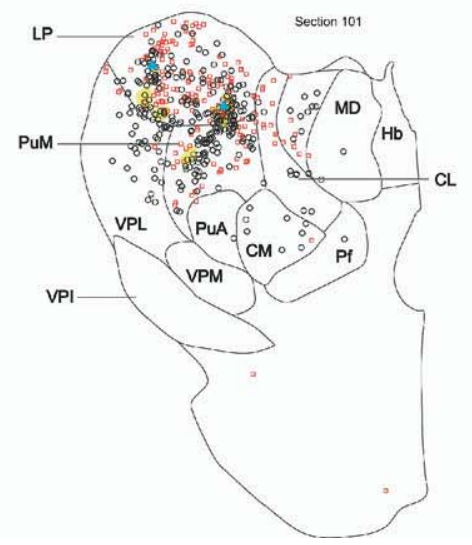

B

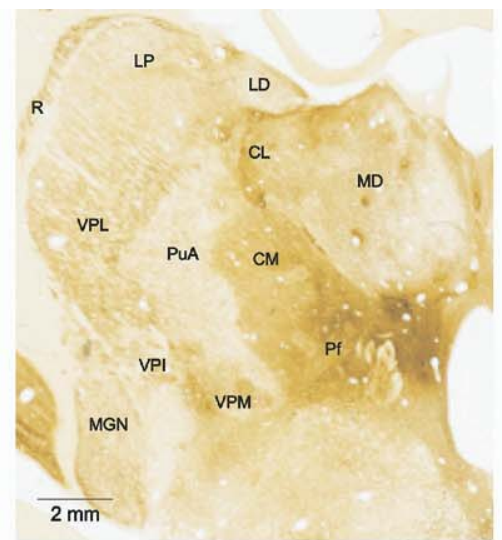

D

F
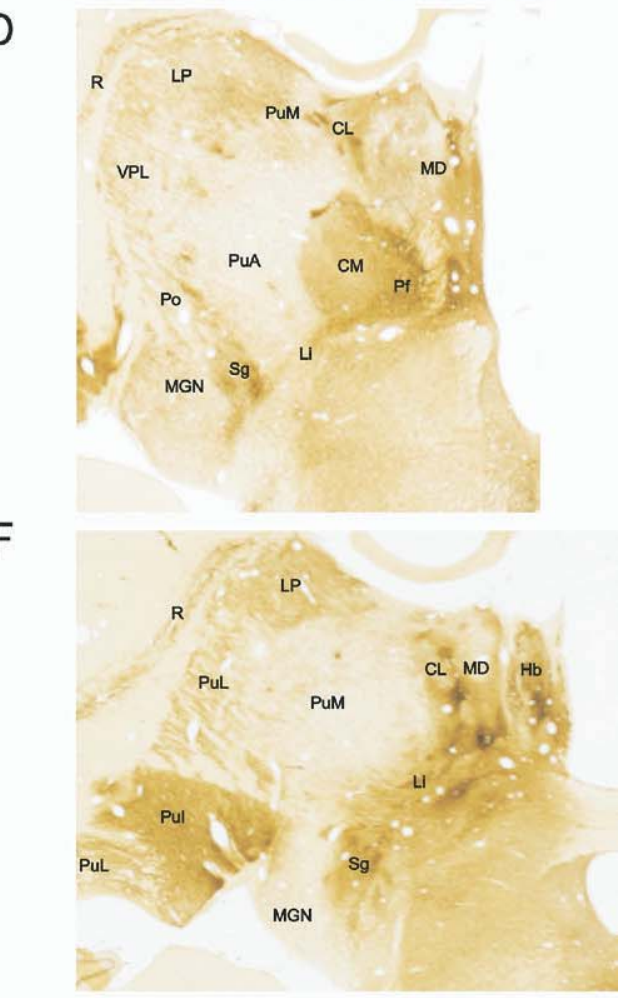

$\mathrm{H}$

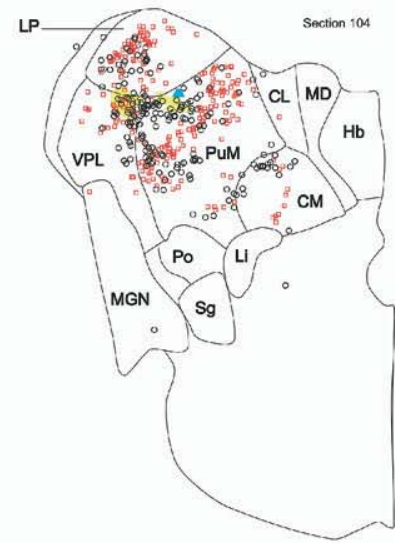

Fig. 5. Additional sections to illustrate more precisely the distribution of $C T$ and $T C$ labeling in the caudal part of the thalamus (A, C and $E$ for MK2; $G$ and $H$ for MK1). Photomicrographs of sections adjacent to those in $A, C$ and $E$, and stained for $A C h E$, are shown in $B, D$ and $F$. Same conventions as in Figs. 3 (panels $\mathrm{G}, \mathrm{H}$ ) and 4 (A, C, E). 
A

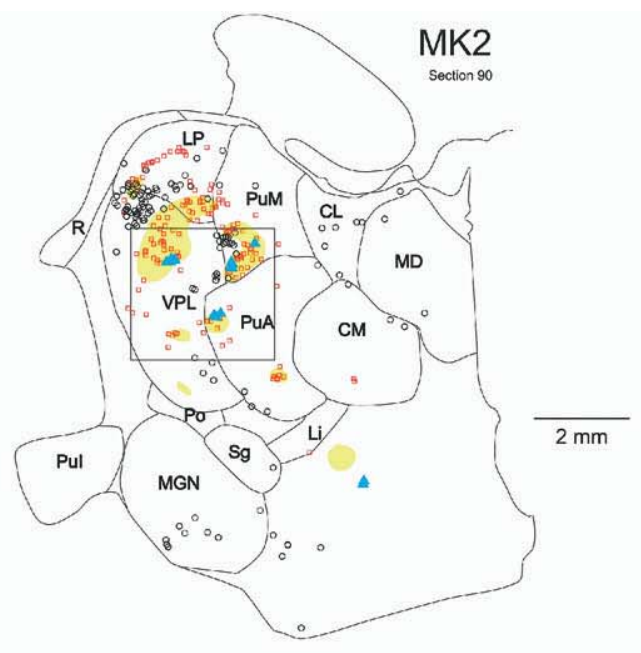

C

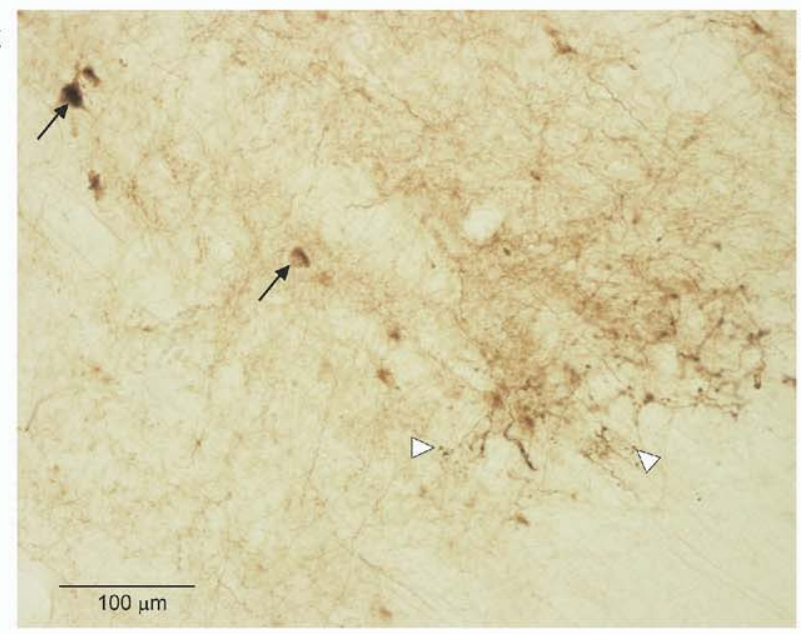

E

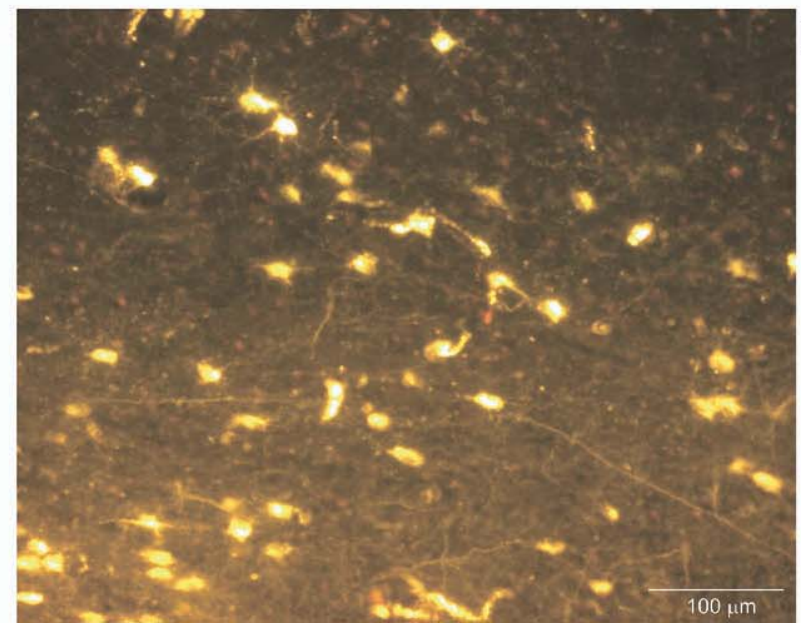

B
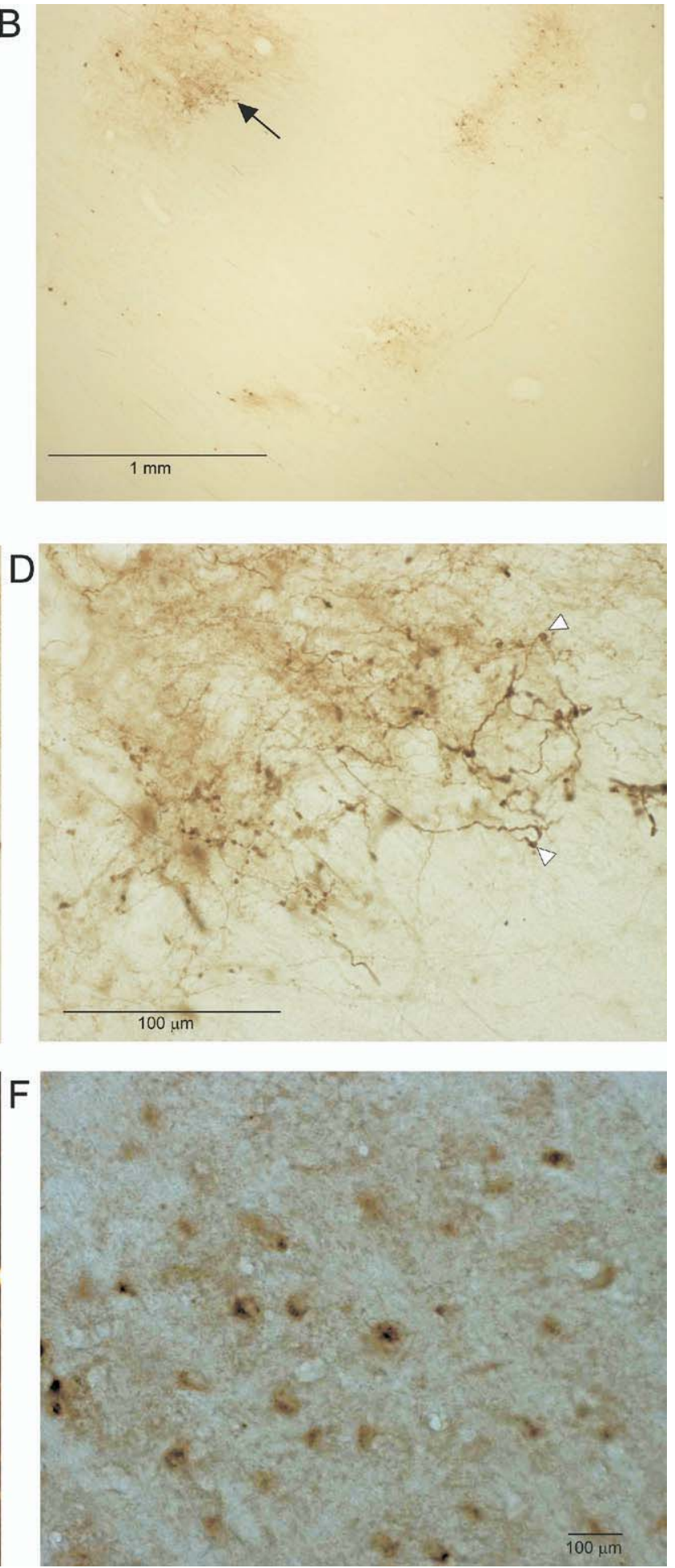

Fig. 6. Photomicrographs of $C T$ endings $(B, C$ and $D$ ). Small $C T$ endings correspond to the yellow territories shown in panel $A$ (same conventions as in Fig. 3). Examples of giant endings are shown with white arrowheads ( $C$ and $D)$. Panel $B$ corresponds to the square area in panel $A$. Panels $C$ and $\mathrm{D}$ are high magnification of the region pointed by an arrow in panel B. BDA-labeled TC neurons are pointed by black arrows in panel C. Panel E illustrates retrogradely labeled neurons in the thalamus after injection of FR in area PEa (MK1), and panel F, labeled neurons in the thalamus as a result of WGA injection in area PE (MK2).

7B and 7D, with the main thalamic nuclei of origin being LP ( $57 \%$ of TC connections for MK1 and $36 \%$ for MK2), PuM
(14\% for MK1 and 10\% for MK2), VPL (11\% for MK1 and $12 \%$ for MK2) and MD (5\% for MK1 and $9 \%$ for MK2). 

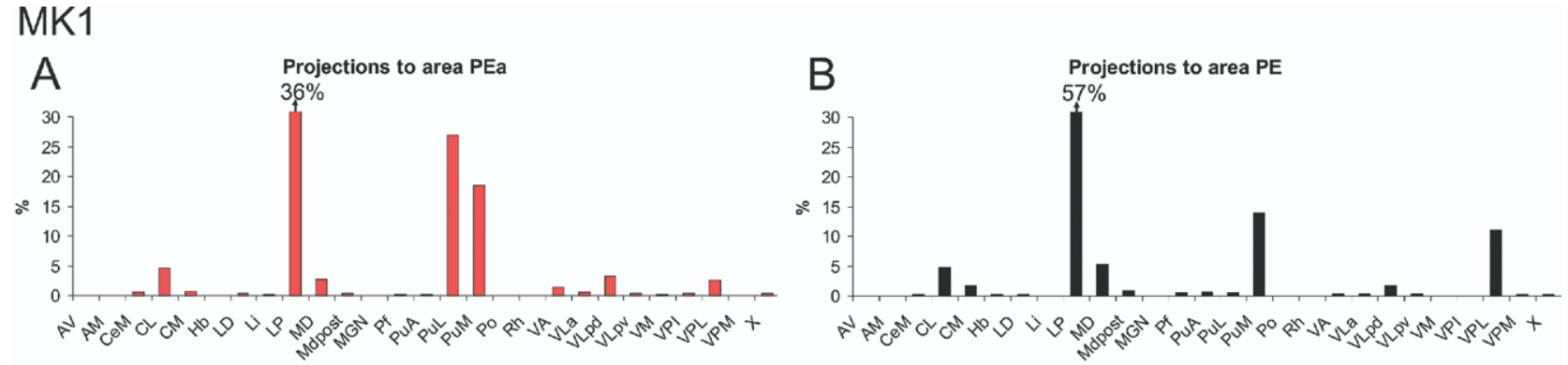

\section{MK2}
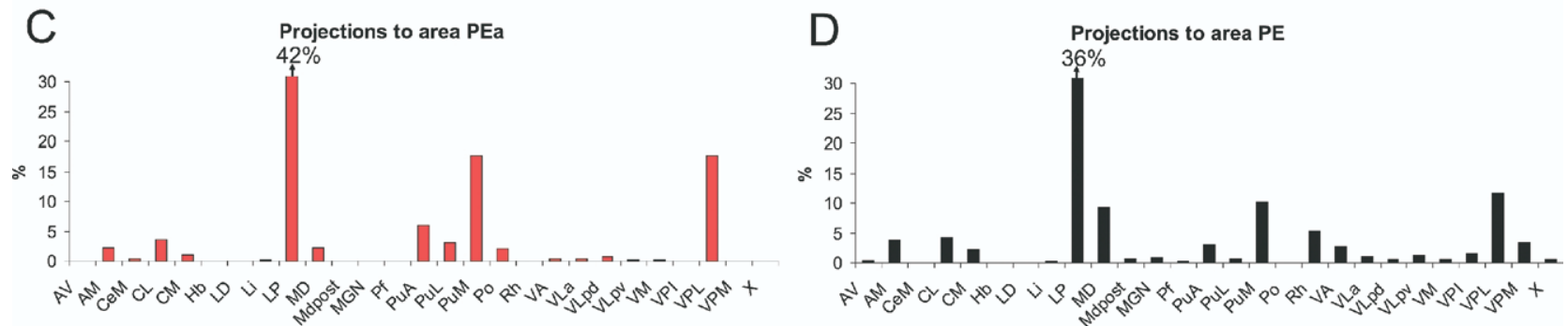

\section{E}

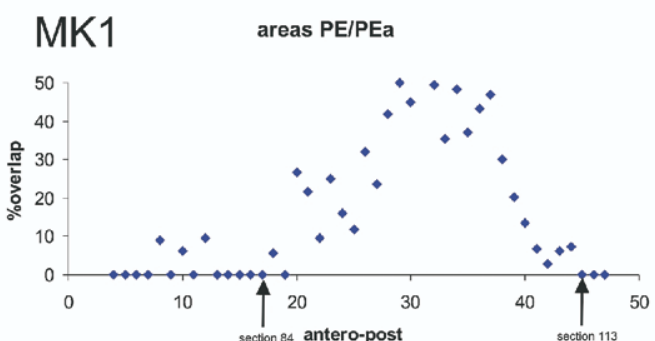

$\mathrm{F}$

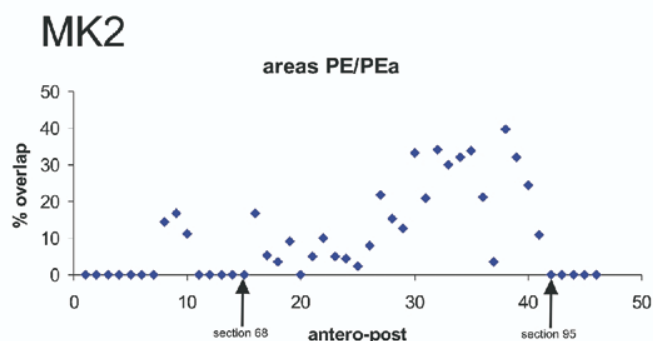

Fig. 7. Quantitative distribution of TC projections directed to areas PE and PEa. (A-D) Histograms of the percentages of labeled cells in each thalamic nucleus with respect to the total number of cells in thalamus labeled by each of the injected cortical areas. Panels A and B are histograms for TC projections to areas PEa and PE, respectively, for MK1. Panels $C$ and D are histograms for TC projections to areas PEa and PE, respectively, for MK2. For each histogram, the sum of all bins is $100 \%$. (E, F) Overlap versus segregation of TC projections to areas PE and PEa, plotted as a function of the rostro-caudal location of the corresponding section of the thalamus in MK1 (E) and MK2 (F). Sections are numbered 1-50, from rostral to caudal, and intervals between sections are $200 \mu \mathrm{m}$. Arrows indicate the position of sections illustrated in Fig. 3 for panel E and in Fig. 4 for panel F.

Fewer neurons in CL, CM and PuA (for MK2) project to area PE. Overall, the main thalamic nucleus projecting to both areas PEa and PE is clearly LP accompanied by significant input coming from PuM and VPL. Moreover, as for CT connections, TC projections to areas PEa and PE are densest in the caudal thalamic nuclei.

\section{Overlap versus segregation of TC projections to areas PE and PEa}

The distribution of retrogradely labeled neurons after multiple tracer injections in areas PE and PEa, as represented in Figs. 3, 4 and 5, shows at first glance that the degree of overlap versus segregation between thalamic projections to these two parietal cortical areas varies across thalamic nuclei (or groups of nuclei). In order to estimate more quantitatively the degree of overlap versus segregation of these TC projections, an index of overlap between two populations of labeled neurons was calculated for the two tracers injected in areas PE and PEa in each monkey (as explained in Experimental Procedures). For methodological reasons, this calculation was applied to the entire thal- amus, without considering nuclear boundaries. Fig. 7E and $F$ displays the degree of overlap between the TC connections of areas PE and PEa along the rostrocaudal axis of thalamus. The average percentage of overlap of the TC projections directed to areas $\mathrm{PE}$ and $\mathrm{PEa}$ on the entire thalamus is $20 \%$ for MK1 and $11 \%$ for MK2. However, considering only the caudal part of thalamus (from the rostral limit of LP and VPL nuclei to the caudal extent of PuM), the average overlap rises up to $36 \%$ for MK1 and $26 \%$ for MK2.

\section{DISCUSSION}

\section{Location of injection sites}

Two distinct sub-areas of the posterior parietal cortex were injected in the superior parietal lobule. The region injected in the anterior bank of IPS corresponds to area PEa (named also area $5 \mathrm{v}$ ) whereas the region injected in the anterior bank surface of IPS is area PE (named also area $5 d$ ), as referred to in previous studies (Pandya and Seltzer, 1982; Seltzer and Pandya, 1984, 1986; Matelli et al., 1998). 


\section{Topography of TC projections}

The distributions and relative proportions of labeled cells in different thalamic nuclei after tracer injections in areas $\mathrm{PEa}$ and PE were quantified in the two monkeys (Fig. 7). There is variability between MK1 and MK2, especially for the projections to area PEa originating from PuL and VPL. This variability is probably due to some variations of injection sites: in contrast to the injection in area PEa for MK1, which was restricted to this area, the injection site for MK2 was slightly more caudal than for MK1 and possibly spread to area MIP. It is also possible that there was an uptake of the tracer in the area VIP in the fundus of IPS for the injection in PEa for MK1. These spreadings could explain that retrograde labeling was higher in PuL but less dense in VPL for MK1 than for MK2 (see Baleydier and Morel, 1992).

Overall, our main findings are that TC projections to area PEa originate mainly from LP, PuM and VPL and from LP, PuM, VPL and MD to area PE. Less dense, but stable across monkeys, TC projections to areas PE and PEa originate from $\mathrm{CL}$. In the monkey, few previously available retrograde tracing studies for area $\mathrm{PE}$ yielded contrasting results: whereas Pearson et al. (1978) and Jones et al. (1979) found projections originating only from $L P, C L$ and PuA, Miyata and Sasaki (1983) as well as Avendano et al. (1990) observed major projections from LP and PuA, accompanied by moderate to heavy projections from $\mathrm{VL}, \mathrm{CL}$, PuM, VPL, CM and a modest contribution from MD, VPI and PuL. In general agreement, Pons and Kaas (1985) observed retrograde labeling in VPL, LP and PuA after injection in area 5. Another study (Acuna et al., 1990) reported TC projections to area 5 originating from different thalamic nuclei such as LP, PuL, PuA and very few from PuM, MD and CM, but not from PuL. Our results (Fig. 7) are in general agreement with Miyata and Sasaki (1983), Avendano et al. (1990) and Acuna et al. (1990) with however some discrepancies in the respective weight of the projections, such as sparse labeling in PuA and a quite dense labeling in PuM (thus diverging from Acuna et al., 1990). These quantitative differences in origin of TC projections may be explained by the differences in the precise location of the injection sites. Indeed, the injection sites performed by Avendano et al. (1990) were more medial than our injections. The injection sites reported by Pearson et al. (1978) were more rostral than our injections. Moreover, in contrast to these studies, we performed several injections (up to seven) in area PE. Compared with the injection sites performed by Acuna et al. (1990), our injection sites clearly covered a larger rostro-caudal extent.

The present study and others (Miyata and Sasaki, 1983; Pons and Kaas, 1985; Avendano et al., 1990) on TC connections of area 5 show clearly the existence of projections from VPL to area 5 . The ventroposterior nucleus has been shown to have a somatotopic organization (Welker, 1974; Kaas et al., 1984; Kaas and Pons, 1988; Rausell et al., 1998). In the present study in particular, retrograde labeling was found in VPL after injections in areas PE and PEa dorsally. The labeled part of VPL cor- responds most likely to trunk representation. As we did not perform an injection of two different tracers in the same area (areas PE or PEa) in the same animal, the present data are not suitable to address the issue of the somatotopic representation in these sub-regions of area 5 . The present study demonstrates that areas PE and PEa share several common properties regarding the origin of their TC projections. At the level of corticocortical connections, many studies have investigated the cortical network of areas PE and/or PEa. First of all the two areas are interconnected. In addition, both areas have reciprocal corticocortical connections with the primary motor cortex (Leichnetz, 1986), area 6 (in particular SMA-proper) (Petrides and Pandya, 1984; Luppino et al., 1993; Matelli et al., 1998), frontal eye field (FEF) (Stanton et al., 1995, 2005) and with areas 1, 2 and 3a (DeFelipe et al., 1986; Burton and Fabri, 1995) (see also Pons and Kaas, 1986, for review). Areas PE and PEa have also their own separate network: area PE projects to dorsal area 4 (Petrides and Pandya, 1984) whereas the motorcortical larynx area projects to area PEa (Simonyan and Jürgens, 2002). Moreover, area PEa receives input from the ventral and caudal superior parietal lobule (areas PE and PEc) as well as from the medial surface of the parietal lobule (area PGm) (Seltzer and Pandya, 1986). Finally, area PEa has a small projection to the parieto-occipital area V6A (Caminiti et al., 1999; Marconi et al., 2001), while area PE is reciprocally connected with area PG (7a) (Neal et al., 1990). Overall, the corticocortical connections of areas PE and PEa are established predominantly with motor and somatosensory areas of the neocortex.

On a functional point of view, microstimulation of posterior parietal cortical areas, including area 5, evokes ecologically relevant behaviors including defensive movements, hand to mouth movements, facial grimaces and aggressive movements (Cooke et al., 2003; Stepniewska et al., 2005). Furthermore, an electrophysiological study (Acuna et al., 1990) in area PE and in the LP and pulvinar nucleus $(\mathrm{Pu})$ of thalamus in a monkey executing reaching limb movements showed that the majority of cells in area PE were classified as reaching related cells. The lateralposterior and pulvinar cells were also reaching related neurons but depended more on the intentionality of movement than cells in area PE. Parietal ablations in monkeys have confirmed involvement of area PE in reaching movements (Ettlinger and Kalsbeck, 1962; Lamotte and Acuna, 1978). Area PE and the LP-Pu complex have reciprocal connections. In line with the present study, area PE was reported to receive its thalamic input mainly from the LP-Pu complex (see also Trojanowski and Jacobson, 1976; Yeterian and Pandya, 1985). A cooperation between area PE and the Pu during goal-directed movements is thus likely. Moreover, in monkey, lesions in area PE and area 7 decreased the precision of visually guided movement (Fabre-Thorpe et al., 1986). On the other hand, area PEa has been shown to play a role in eye-hand coordination during reaching movements (Marconi et al., 2001) and has connections with parietal areas V6A and PEc, as well as with motor cortex. In conclusion, areas PE and 
PEa, classically known as sensorimotor association areas, participate also in the control of visually guided movements (Pandya and Seltzer, 1982; McGuire et al., 1989) and, in particular, area PE is involved in egocentric representation of reaching in monkeys (Acuna et al., 1990; for review, see Lacquaniti et al., 1995; Kalaska, 1996). Moreover, in a recent electrophysiological study in area 5 (Gardner et al., 2006) conducted on macaque monkeys trained to a prehension task, it was found that the activity of $88 \%$ of task-related neurons was influenced during approach, contact and grasp of the object in area 5 .

As described above, the thalamic inputs to areas PE and PEa originate principally from LP. Such predominant projection from LP to area 5 has been reported earlier by Pons and Kaas (1985). The nucleus LP is also closely interconnected with area 7 (Burton and Jones, 1976), including the three visually mapped dorsomedial, medial and posterior parietal areas (Graham et al., 1979). Another substantial source of input to areas PE and PEa appears to be the nucleus VPL, known as the main somatosensory relay (Kaas and Pons, 1988, for review). The third main input to areas PE and PEa comes from PuM. PuM projects also to the cortical areas FEF, V4, MT, infero-temporal area (IT), area 7 , the belt and the parabelt of the auditory cortex, the prefrontal cortex and the cingular cortex (see Stepniewska, 2004, for review) and could be implicated in the control of visually guided movements (Fabre-Thorpe et al., 1986). Otherwise, rostral portions of the dorsal thalamus, generally associated with motor processing (Vitek et al., 1994, 1996), also provide input to the areas PE and PEa, as well as MD (particularly to area PE), CL and PuA. Thus, the present study confirms that areas $\mathrm{PE}$ and $\mathrm{PEa}$ are sensorimotor areas in general, with a particular role in visually guided movements.

\section{Overlap versus segregation of TC projections to areas PE and PEa}

The overlap between zones of origin of the TC projections to areas PE and PEa is considerable, especially in the caudal thalamus. Indeed, both areas receive their main thalamic inputs from the caudal thalamic nuclei LP, PuM and VPL. In addition, but to a lesser extent, areas PE and $\mathrm{PEa}$ receive both TC inputs from the thalamic nuclei PuL, $C L$ and MD. On a quantitative point of view, the degree of overlap of the TC projections to areas PE and PEa amounts to $36 \%$ for the caudal thalamic nuclei, which can be compared, using the same criteria, to that between TC projections to the rostral (PMv-r) and caudal (PMv-c) parts of ventral premotor cortex $(38 \%)$ or to the rostral (PMd-r) and caudal (PMd-c) parts of dorsal premotor cortex (39\%) in the rostral thalamus (Morel et al., 2005). For further comparison, the TC overlap found here for areas PE and PEa $(36 \%)$ is higher than that found for pre-SMA and SMA-proper (20\%, see Morel et al., 2005).

The large overlap of the zones of origin of the TC projections to areas $\mathrm{PE}$ and $\mathrm{PEa}$ opens the possibility that a single TC neuron may send a diverging projection to both $\mathrm{PE}$ and PEa. Such diverging TC projection would be represented by double-labeled cells. Unfortunately, this issue could not be addressed here as the combination of the two tracers in the same animal (FR and BDA in MK1; WGA and BDA in MK2) did not allow the visualization of double labeling as each marker was revealed and analyzed on distinct series of sections.

\section{Morphology and topography of CT endings}

The dual pattern of CT projections refers to the presence of small and giant endings, as well as to the pattern of arrangement of giant endings. The present study is the first report on the distribution of small and giant $C T$ endings originating from the posterior parietal areas PE and PEa. Small endings were more widely distributed and numerous than giant endings, in line with previous observations for the motor, auditory, somatosensory and visual systems (Rouiller and Welker, 2000 for review). Thus, the present data extend the concept of a dual morphology and topography of the CT terminals to the associative parietal cortex. In monkeys, the duality of small and giant CT endings is well established concerning the primary cortical areas, such as M1 (Rouiller et al., 1998), V1 (Rockland, 1996), 3b (Darian-Smith et al., 1999), considered as S1 "proper" (Kaas, 1983; Kaas and Pons, 1988) and A1 (Rouiller and Durif, 2004). The dual CT projection was also shown for area 1, area $7 \mathrm{a}$ and area Opt, the caudalmost area of the intraparietal lobe (Darian-Smith et al., 1999; Taktakishvili et al., 2002), and therefore the present study confirms the existence of a dual pattern of CT endings also for nonprimary parietal cortical areas, area 5 in the present case.

In terms of topographical organization of CT connections, our data show a distribution of both small and giant endings from area $P E a$ in the thalamic nuclei $L P, V P L$, PuA, PuM, MD and PuL and from area PE in LP, PuM, and VPL. These results are in agreement with previous study of the CT connections of posterior parietal regions (Yeterian and Pandya, 1985).

\section{Reciprocity of CT and TC projections}

In general, most studies report that TC and CT systems of projection largely overlap in thalamus, thus implying that the territories formed by CT small and giant endings generally match the clusters of TC neurons. This general match of CT terminals and TC neuronal clusters was reported for the primary auditory cortex in the cat (Winer and Larue, 1987) as well as in motor cortex in monkeys (Rouiller et al., 1998, 2003). However, more detailed observations provided evidence for some mismatch, although non-overlapping territories were spatially relatively restricted. A non-strict reciprocity of CT and TC projections has also been observed for the posterior parietal cortex (Darian-Smith et al., 1999 and Taktakishvili et al., 2002). At first sight, TC and CT matching zones are also present here as a result of BDA injection in areas PE and PEa, more so after injection in area $\mathrm{PEa}$ (Fig. 3; Fig. 5 panels $A$, $C$ and $E$ ) than in area PE (Fig. 4; Fig. 5 panels $G$ and $H$ ). However, in both monkeys, although more prominently in MK1, there are large zones of thalamus with labeled TC neurons but devoid of anterograde labeling (outside the yellow zones in Figs. 3-5). In other words, the TC territo- 
ries appear larger than the CT ones, a mismatch that seems to be more pronounced for areas PE and PEa than for other cortical areas such as primary auditory (Rouiller and Durif, 2004), primary motor and premotor cortical areas (Rouiller et al., 1998, 2003). Each cortical area was injected at multiple sites, which cover the different cortical layers if we considered all these sites (see Fig. 2). However, it cannot be excluded that the BDA injections did not cover an equal volume of cortex in layers III/IV, targets of $\mathrm{TC}$ projection, and in layers $\mathrm{V} / \mathrm{VI}$, sources of $\mathrm{CT}$ projections. This could be a part of the explanation of the mismatch. Another possible reason for substantial mismatch is that, as apparent in Fig. 2, the BDA injection may not be restricted to the same single cortical columns for the different layers (III/IV versus V/VI) and therefore distinct thalamic territories will be labeled for the TC and CT projection thus introducing mismatch.

\section{Functional significance of the small and giant endings CT projections}

Different roles for the CT projections were proposed in previous studies (Welker et al., 1988; Rouiller and Welker, 1991; Rouiller et al., 2003) and reviewed extensively by Rouiller and Welker (2000) and Sherman and Guillery (2005). Small CT terminals (originating from layer VI neurons) are typically present in the projection serving the feedback control of cerebral cortex on the thalamic nucleus from which it receives its main inputs and is typically associated to a modulatory function. In contrast, giant terminals (originating from layer $\mathrm{V}$ neurons) are involved in feedforward projections by which activity from a cortical area is distributed, via the thalamus, to other parts of cerebral cortex. Functionally, the giant endings CT projection is considered as a driving activity. This functional disparity (modulator versus driver) is supported by electrophysiological data derived from in vitro preparations ( $\mathrm{Li}$ et al., 2003; Reichova and Sherman, 2004). The most common EPSPs, produced by stimulation of layer VI neurons, showed a graded, long latency response with slower rise time than the less frequently encountered EPSPs (arising from layer $V$ stimulation) characterized by large, all or none response with shorter latency.

The cross-species and cross-systems comparison reveals differences in the mode of feedforward projections, which may be involved in the activation of other parts of the same cortical area or of other, remote cortical areas. It is interesting to note that at the level of the neocortex, the cortico-cortical feedback connections have also been described as more widespread than feedforward ones (Maunsell and Van Essen, 1983). Like the feedforward connections in the cortex, the CT projections formed by thick fibers and terminating with giant endings represent the support for fast, secure, transthalamic interactions between distant cortical regions (see also Feig and Harting, 1998; Sherman and Guillery, 2002; van Horn and Sherman, 2004). Although, one may expect the giant CT endings to be strictly segregated from the small CT endings, thus corresponding to a clear functional separation of the driving and modulatory influences of the cortex onto the thalamus, it is usually not the case. Indeed, in general, giant CT endings co-localize with some small CT endings, an observation that was also true here for area 5 in the thalamic nuclei LP, PuM, PuA and VPL. These thalamic nuclei send TC projections to cortical areas remote from area 5 , such as somatosensory areas $3 b$ and 1 , area 7 , the lateral intraparietal area (LIP), the prefrontal cortex, the FEF, the IT, the auditory cortex (parabelt) and the motor cortex. If the CT and TC territories in the thalamus overlap, they represent a possible an anatomical support for rapid and secure transthalamic transmission of information from area 5, via LP, PuA, PuM and VPL to the abovementioned cortical areas, in other words to remote cortical regions in the parietal, temporal and frontal lobes.

\section{CONCLUSION}

In conclusion, the present study supports the notion that area 5 in the macaque monkey comprises two cytoarchitectonally distinct sub-areas, PE and PEa, as assessed in Nissl- and SMI-32 stained material. The areas PE and PEa have largely comparable patterns of TC and CT projections, mainly with the caudal thalamus, more specifically with the nuclei LP, PuM and VPL. The thalamic territories projecting to areas PE and PEa overlap to a significant extent, as much as those directed to the two subdivisions of the dorsal premotor cortex (PMd-c and PMd-r) or the ventral premotor cortex (PMv-c and PMv-r). Finally, the present TC and CT projections observed for the areas PE and PEa represent a possible anatomical substrate for transthalamic, multisensory and sensorimotor integration processes involving area 5 in primates.

Acknowledgments-The authors wish to thank technical assistance of Véronique Moret, Georgette Fischer, Françoise Tinguely, Christine Roulin and Veronika Streit (histology), Josef Corpataux, Bernard Bapst, Laurent Bossy and Bernard Morandi (animal housekeeping), Laurent Monney (informatics).

Grant Sponsors: Swiss National Science Foundation, grants No. 31-61857.00, 310000-110005 (E.M.R.), Novartis Foundation; The Swiss National Science Foundation Centre of Competence in Research (NCCR) on "Neural plasticity and repair."

\section{REFERENCES}

Acuna C, Cudeiro J, Gonzalez F, Alonso JM, Perez R (1990) Lateralposterior and pulvinar reaching cells-comparison with parietal area 5a: a study in behaving Macaca nemestrina monkeys. Exp Brain Res 82:158-166.

Avendano C, Stepniewska I, Rausell E, Reinoso-Suarez F (1990) Segregation and heterogeneity of thalamic cell populations projecting to superficial layers of posterior parietal cortex: a retrograde tracer study in cat and monkey. Neuroscience 39(3):547-559.

Bajo VM, Rouiller EM, Welker E, Clarke S, Villa AEP, de Ribaupierre Y, de Ribaupierre F (1995) Morphology and spatial distribution of corticothalamic terminals originating from the cat auditory cortex. Hear Res 83:161-174.

Baleydier C, Morel A (1992) Segregated thalamocortical pathways to inferior parietal and inferotemporal cortex in macaque monkey. Vis Neurosci 8:391-405.

Benes FM, Lange N (2001) Two-dimensional versus three-dimensional cell counting: a practical perspective. Trends Neurosci 24:11-17. 
Burton H, Fabri M (1995) Ipsilateral intracortical connections of physiologically defined cutaneous representations in areas $3 \mathrm{~b}$ and 1 of macaque monkeys: projections in the vicinity of the central sulcus. J Comp Neurol 355(4):508-538.

Burton H, Jones EG (1976) The posterior thalamic region and its cortical projection in New World and Old World monkeys. J Comp Neurol 168:520-523.

Bourassa J, Deschênes M (1995) Corticothalamic projections from the primary visual cortex in rats: a single fiber study using biocytin as an anterograde tracer. Neuroscience 66(2):253-263.

Bourassa J, Pinault D, Deschênes M (1995) Corticothalamic projections from the cortical barrel field to the somatosensory thalamus in rats: A single-fibre study using biocytin as an anterograde tracer. Eur J Neurosci 7:19-30.

Caminiti R, Genovesio A, Marconi B, Mayer AB, Onorati P, Ferraina S, Mitsuda T, Giannetti S, Squatrito S, Maioli MG, Molinari M (1999) Early coding of reaching: frontal and parietal association connections of parieto-occipital cortex. Eur J Neurosci 11(9):3339-3345.

Campbell MJ, Morrison JH (1989) Monoclonal antibody to neurofilament protein (SMI-32) labels a subpopulation of pyramidal neurons in the human and monkey neocortex. J Comp Neurol 282(2):191-205.

Cappe C, Barone P, Rouiller EM (2005) Anatomical support for a role of the thalamo-cortical pathway in the multisensory integration in monkeys. 35th Meeting of the Society for Neuroscience (SFN), Washington (USA), abstr. 617.9-T.

Cooke DF, Taylor CS, Moore T, Graziano MS (2003) Complex movements evoked by microstimulation of the ventral intraparietal area. Proc Natl Acad Sci U S A 100(10):6163-6168.

Darian-Smith C, Darian-Smith I, Cheema SS (1990) Thalamic projections to sensorimotor cortex in the macaque monkey: use of multiple retrograde fluorescent tracers. J Comp Neurol 299:17-46.

Darian-Smith C, Tan A, Edwards S (1999) Comparing thalamocortical and corticothalamic microstructure and spatial reciprocity in the macaque ventral posterolateral nucleus (VPLc) and medial pulvinar. J Comp Neurol 410:211-234.

DeFelipe J, Conley M, Jones EG (1986) Long-range focal collateralization of axons arising from corticocortical cells in monkey sensory-motor cortex. J Neurophysiol 6(12):3749-3766.

Ettlinger G, Kalsbeck JE (1962) Changes in tactile discrimination and in visual reaching after successive and simultaneous bilateral posterior parietal ablations in the monkey. J Neurol Neurosurg Psychiatry $25: 256-268$.

Fabre-Thorpe M, Vievard A, Buser P (1986) Role of the extra-geniculate pathway in visual guidance. II. Effects of lesioning the pulvinar-lateral posterior thalamic complex in the cat. Exp Brain Res 62(3):596-606.

Feig S, Harting JK (1998) Corticocortical communication via the thalamus: Ultrastructural studies of corticothalamic projections from area 17 to the lateral posterior nucleus of the cat and inferior pulvinar nucleus of the owl monkey. J Comp Neurol 395:281-295.

Gardner EP, Babu KS, Reitzen SD, Ghosh S, Brown AM, Chen J, Hall AL, Herzlinger M, Kohlenstein JB, Ro JY (2006) Neurophysiology of prehension: I. Posterior parietal cortex and object-oriented hand behaviors. J Neurophysiol, online; doi: 10.1152/jn.00558.2006.

Geneser-Jensen FA, Blackstad TW (1971) Distribution of acetyl cholinesterase in the hippocampal region of the guinea pig. I. Entorhinal area, parasubiculum, and presubiculum. Zellforsch Mikrosk Anat 114(4):460-481

Geuna S (2000) Appreciating the difference between design-based and model-based sampling strategies in quantitative morphology of the nervous system. J Comp Neurol 427:333-339.

Giguere M, Goldman-Rakic PS (1988) Mediodorsal nucleus: Areal, laminar, and tangential distribution of afferents and efferents in the frontal lobe of rhesus monkeys. J Comp Neurol 277:195-213.

Graham J, Lin CS, Kaas JH (1979) Subcortical projections of six visual cortical areas in the owl monkey, Aotus trivirgatus. J Comp Neurol 187:557-580.
Guillery RW (1995) Anatomical evidence concerning the role of the thalamus in corticocortical communication: A brief review. J Anat 187:583-592.

Hatanaka N, Tokuno H, Hamada I, Inase M, Ito Y, Imanishi M, Hasegawa N, Akazawa T, Nambu A, Takada M (2003) Thalamocortical and intracortical connections of monkey cingulate motor areas. J Comp Neurol 462:121-138.

Hoogland PV, Welker E, van der Loos H (1987) Organization of the projections from barrel cortex to thalamus in mice studied with Phaseolus vulgaris-leucoagglutinin and HRP. Exp Brain Res 68:73-87.

Hoogland PV, Wouterlood FG, Welker E, van der Loos H (1991) Ultrastructure of giant and small thalamic terminals of cortical origin: A study of the projections from the barrel cortex in mice using Phaseolus vulgaris leuco-agglutinin (PHA-L). Exp Brain Res 87:159-172.

Jones EG, Wise SP, Coulter JD (1979) Differential thalamic relationships of sensory-motor and parietal cortical fields in monkeys. J Comp Neurol 183:833-882.

Jones EG, Hendry SHC (1989) Differential calcium binding protein immunoreactivity distinguishes classes of relay neurons in monkey thalamic nuclei. Eur J Neurosci 1:222-246.

Jones EG (1990) Correlation and revised nomenclature of ventral nuclei in the thalamus of human and monkey. Stereotact Funct Neurosurg 54-55:1-20.

Jones EG (1985) The thalamus. New York: Plenum Press.

Kaas JH, Nelson RJ, Dykes RW, Merzenich MM (1984) The somatotopic organization of the ventroposterior thalamus of the squirrel monkey, Saimiri sciuereus. J Comp Neurol 226:111-140.

Kaas JH, Pons TP (1988) The somatosensory system of primates. In: Comparative primate biology, Vol. 4 (Steklis HP, Erwin J, eds), pp 421-468. New York: Liss.

Kaas JH (1983) What, if anything, is S-I? The organization of the "first somatosensory area" of cortex. Physiol Rev 63:206-231.

Kakei S, Na J, Shinoda Y (2001) Thalamic terminal morphology and distribution of single corticothalamic axons originating from layers 5 and 6 of the cat motor cortex. J Comp Neurol 437:170-185

Kalaska JF (1996) Parietal cortex area 5 and visuomotor behavior. Can J Physiol Pharmacol 74:483-498.

Kelly JP, Wong D (1981) Laminar connections of the cat's auditory cortex. Brain Res 212:1-15.

Lacquaniti F, Guigon E, Bianchi L, Ferraina S, Caminiti R (1995) Representing spatial information for limb movement: Role of area 5 in the monkey. Cereb Cortex 5:391-409.

Lamotte RH, Acuna C (1978) Defects in accuracy of reaching after removal of posterior parietal cortex in monkeys. Brain Res 139: 309-326.

Lavenex P, Steele MA, Jacobs LF (2000) The seasonal pattern of cell proliferation and neuron number in the dentate gyrus of wild adult eastern grey squirrels. Eur J Neurosci 12:643-648.

Leichnetz GR (1986) Afferent and efferent connections of the dorsolateral precentral gyrus (area 4, hand/arm region) in the macaque monkey, with comparisons to area 8. J Comp Neurol 254(4):460-492.

Li J, Guido W, Bickford ME (2003) Two distinct types of corticothalamic EPSPs and their contribution to short-term synaptic plasticity. J Neurophysiol 90:3429-3440.

Liu J, Morel A, Wannier T, Rouiller EM (2002) Origins of callosal projections to the supplementary motor area (SMA): A direct comparison between pre-SMA and SMA-proper in macaque monkeys. J Comp Neurol 443:71-85

Luppino G, Matelli M, Camarda R, Rizzolatti G (1993) Corticocortical connections of area F3 (SMA-proper) and area F6 (pre-SMA) in the macaque monkey. J Comp Neurol 338:114-140.

Marconi B, Genovesio A, Battaglia-Mayer A, Ferraina S, Squatrito S, Molinari M, Lacquaniti F, Caminiti R (2001) Eye-hand coordination during reaching. I. Anatomical relationships between parietal and frontal cortex. Cereb Cortex 11:513-527. 
Matelli M, Luppino G, Fogassi L, Rizzolatti G (1989) Thalamic input to inferior area 6 and area 4 in the macaque monkey. J Comp Neurol 280:468-488.

Matelli M, Govoni P, Galletti C, Kutz DF, Luppino G (1998) Superior area 6 afferents from the superior parietal lobule in the macaque monkey. J Comp Neurol 402:327-352.

Maunsell JHR, Van Essen DC (1983) The connections of the middle temporal visual area (MT) and their relationship to a cortical hierarchy in the macaque monkey. J Neurosci 3(12):2563-2586.

McGuire PK, Hockfield S, Goldman-Rakic PS (1989) Distribution of cat-301 immunoreactivity in the frontal and parietal lobes of the macaque monkey. J Comp Neurol 288(2):280-296.

Miyata M, Sasaki K (1983) HRP studies on thalamocortical neurons related to the cerebellocerebral projection in the monkey. Brain Res 274:213-224.

Morel A, Liu J, Wannier T, Jeanmonod D, Rouiller EM (2005) Divergence and convergence of thalamocortical projections to premotor and supplementary motor cortex: a multiple tracing study in macaque monkey. Eur J Neurosci 21(4):1007-1029.

Neal JW, Pearson RCA, Powell TPS (1990) The connections of area PG, $7 a$, with cortex in the parietal, occipital and temporal lobes of the monkey. Brain Res 532:249-264.

Ojima H, Murakami K, Kishi K (1996) Dual termination modes of corticothalamic fibers originating from pyramids of layers 5 and 6 in cat visual cortical area 17. Neurosci Lett 208:57-60.

Ojima H (1994) Terminal morphology and distribution of corticothalamic fibers originating from layers 5 and 6 of cat primary auditory cortex. Cereb Cortex 4(6):646-663.

Olszewski J (1952) The thalamus of Macaca mulatta. An atlas for use with the stereotaxic instrument. Basel: Karger.

Pandya DN, Seltzer B (1982) Intrinsic connections and architectonics of posterior parietal cortex in the rhesus monkey. J Comp Neurol 204:196-210.

Paxinos G, Huang XF, Toga AW (2000) The rhesus monkey brain in stereotaxic coordinates. London: Academic Press.

Pearson RCA, Powell TPS (1978) The cortico-cortical connections to area 5 of the parietal lobe from the primary somatic sensory cortex of the monkey. Proc R Soc Lond B 200:103-108.

Petrides M, Pandya DN (1984) Projections to the frontal cortex from the posterior parietal region in the rhesus monkey. J Comp Neurol 228:105-116.

Pons TP, Kaas JH (1985) Connections of area 2 of somatosensory cortex with the anterior pulvinar and subdivisions of the ventroposterior complex in macaque monkeys. J Comp Neurol 240:16-36.

Pons TP, Kaas JH (1986) Corticocortical connections of area 2 of somatosensory cortex in macaque monkeys: a correlative anatomical and electrophysiological study. J Comp Neurol 15:248(3):313-335.

Rausell E, Bickford L, Manger PR, Woods TM, Jones EG (1998) Extensive divergence and convergence in the thalamocortical projection to monkey somatosensory cortex. J Neurosci 18(11):4216-4232.

Reichova I, Sherman SM (2004) Somatosensory corticothalamic projections: distinguishing drivers from modulators. J Neurophysiol 92:2185-2197.

Rockland KS (1996) Two types of corticopulvinar terminations: round (type 2) and elongate (type 1). J Comp Neurol 368:57-87.

Rouiller EM, Liang F, Moret V, Wiesendanger M (1991) Patterns of corticothalamic terminations following injection of Phaseolus vulgaris leucoagglutinin (PHA-L) in the sensorimotor cortex of the rat. Neurosci Lett 125:93-97.

Rouiller EM, Welker E (1991) Morphology of corticothalamic terminals arising from the auditory cortex of the rat: A Phaseolus vulgarisleucoagglutinin (PHA-L) tracing study. Hear Res 56:179-190.

Rouiller EM, Tanné J, Moret V, Kermadi I, Boussaoud D, Welker E (1998) Dual morphology and topography of the corticothalamic terminals originating from the primary, supplementary motor, and dorsal premotor cortical areas in macaque monkeys. J Comp Neurol 396:169-185.
Rouiller EM, Tanne J, Moret V, Boussaoud D (1999) Origin of thalamic inputs to the primary, premotor, and supplementary motor cortical areas and to area 46 in macaque monkeys: A multiple retrograde tracing study. J Comp Neurol 409:131-152.

Rouiller EM, Welker E (2000) A comparative analysis of the morphology of corticothalamic projections in mammals [review]. Brain Res Bull 53(6):727-741.

Rouiller EM, Wannier T, Morel A (2003) The dual pattern of corticothalamic projection of the premotor cortex in macaque monkeys. Thalamus Related System 2:189-197.

Rouiller EM, Durif C (2004) The dual pattern of corticothalamic projection of the primary auditory cortex in macaque monkey. Neurosci Lett 358:49-52.

Sakata H, Takaoka Y, Kawarasaki A, Shibutani H (1973) Somatosensory properties of neurons in the superior parietal cortex (area 5) of the rhesus monkey. Brain Res 64:85-102.

Schwartz ML, Dekker JJ, Goldman-Rakic PS (1991) Dual mode of corticothalamic synaptic termination in the mediodorsal nucleus of the rhesus monkey. J Comp Neurol 309:289-304.

Seltzer B, Pandya DN (1984) Further observations on parieto-temporal connections in the rhesus monkey. Exp Brain Res 55(2): 301-312.

Seltzer B, Pandya DN (1986) Posterior parietal projections to the intraparietal sulcus of the rhesus monkey. Exp Brain Res 62(3): 459-469.

Sherman SM, Guillery RW (2002) The role of the thalamus in the flow of information to the cortex. Philos Trans R Soc Lond [Biol] 357: 1695-1708.

Sherman SM, Guillery RW (2005) Exploring the thalamus and its role in cortical function. Cambridge: MIT Press.

Simonyan K, Jürgens U (2002) Cortico-cortical projections of the motor cortical larynx area in the rhesus monkey. Brain Res 949:23-31.

Stanton GB, Bruce CJ, Goldberg ME (1995) Topography of projections to posterior cortical areas from the macaque frontal eye fields. J Comp Neurol 353:291-305.

Stanton GB, Friedman HR, Dias EC, Bruce CJ (2005) Cortical afferents to the smooth-pursuit region of the macaque monkey's frontal eye field. Exp Brain Res 165(2):179-192.

Stepniewska I (2004) The pulvinar complex. The primate visual system, pp 53-79. CRC Press.

Stepniewska I, Fang PC, Kaas JH (2005) Microstimulation reveals specialized subregions for different complex movements in posterior parietal cortex of prosimian galagos. Proc Natl Acad Sci U S A 102(13):4878-4883.

Taktakishvili O, Sivan-Loukianova E, Kultas-llinsky K, llinsky IA (2002) Posterior parietal cortex projections to the ventral lateral and some association thalamic nuclei in Macaca mulatta. Brain Res Bull 59:135-150.

Tanné-Gariépy J, Boussaoud D, Rouiller EM (2002) Projections of the claustrum to the primary motor, premotor, and prefrontal cortices in the macaque monkey. J Comp Neurol 454:140-157.

Trojanowski JQ, Jacobson S (1976) Areal and laminar distribution of some pulvinar cortical efferents in rhesus monkey. J Comp Neurol 169(3):371-392.

Tsang YM, Chiong F, Kuznetsov D, Kasarskis E, Geula C (2000) Motor neurons are rich in non-phosphorylated neurofilaments: cross-species comparison and alterations in ALS. Brain Res 861(1):45-58.

van Horn SC, Sherman SM (2004) Differences in projection patterns between large and small corticothalamic terminals. J Comp Neurol 475:406-415.

Vitek JL, Ashe J, DeLong MR, Alexander GE (1994) Physiologic properties and somatotopic organization of the primate motor thalamus. J Neurophysiol 71(4):1498-1513.

Vitek JL, Ashe J, DeLong MR, Kaneoke Y (1996) Microstimulation of primate motor thalamus: somatotopic organization and differential distribution of evoked motor responses among subnuclei [erratum 
appears in J Neurophysiol 1997;77(6):2857]. J Neurophysiol 75(6):2486-2495.

Wannier T, Schmidlin E, Bloch J, Rouiller EM (2005) A unilateral section of the corticospinal tract at cervical level in primate does not lead to measurable cell loss in motor cortex. J Neurotrauma 22(6):703-717.

Welker E, Hoogland PV, van der Loos H (1988) Organization of feedback and feedforward projections of the barrel cortex: a PHA-L study in the mouse. Exp Brain Res 73:411-435.

Welker WI (1974) Principles of organization of the ventrobasal complex in mammals. Brain Behav Evol 7:253-336.
Winer JA, Larue DT (1987) Patterns of reciprocity in auditory thalamocortical and corticothalamic connections: study with horseradish peroxidase and autoradiographic methods in the rat medial geniculate body. J Comp Neurol 257(2):282-315.

Winer JA, Sally SL, Larue DT, Kelly JB (1999) Origins of medial geniculate body projections to physiologically defined zones of rat primary auditory cortex. Hear Res 130:42-61.

Yeterian EH, Pandya DN (1985) Corticothalamic connections of the posterior parietal cortex in the rhesus monkey. J Comp Neurol 237(3):408-426. 